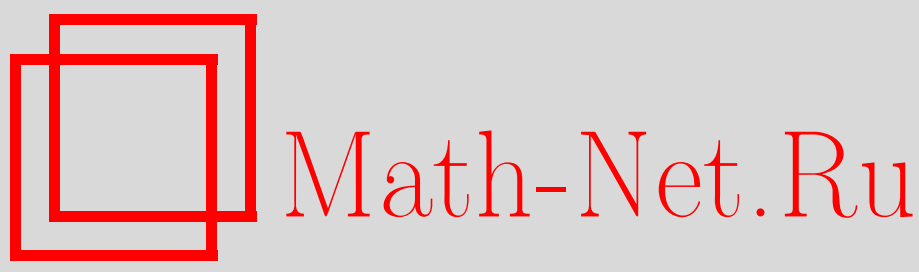

Т. О. Банах, Т. Н. Радул, Топология пространств вероятностных мер, Матем. сб., 1997, том 188, номер 7, 23-46

DOI: https://doi.org/10.4213/sm241

Использование Общероссийского математического портала Math-Net.Ru подразумевает, что вы прочитали и согласны с пользовательским соглашением http://www.mathnet.ru/rus/agreement

Параметры загрузки:

IP : 3.85 .5 .30

26 апреля 2023 г., $16: 57: 24$ 
УДК 515.12

\author{
Т. О. Банах, Т. Н. Радул
}

\title{
Топология пространств вероятностных мер
}

В работе изучается пространство $\widehat{P}(X)$ вероятностных радоновских мер на метрическом пространстве $X$, а также его подпространства $P_{c}(X), P_{d}(X)$ и $P_{\omega}(X)$, состоящие соответственно из непрерьвных мер, дискретных мер и мер с конечными носителями. Доказано, что для любого полно-метризуемого пространства $X$ пространство $\widehat{P}(X)$ гомеоморфин гильбертову пространству. Получена топологическая классификация пар $(\widehat{P}(K), \widehat{P}(X)),\left(\widehat{P}(K), P_{d}(Y)\right)$, $\left(\widehat{P}(K), P_{c}(Z)\right)$, где $K$ - метрический компакт, $X$ - всюду плотное борелевское подмножество в $K, Y$ - всюду плотное $F_{\sigma \delta}$-подмножество $K$, и $Z$ - всюду несчетное всюду плотное борелевское подмножество $K$ достаточно высокого борелевского класса. Найдены необходимые и достаточные условия на пару $(X, Y)$, при которых пара $\left(\widehat{P}(X), P_{\omega}(Y)\right)$ гомеоморфна $\left(l^{2}(A), l_{f}^{2}(A)\right)$.

Библиографоия: 28 названий.

\section{Введение}

Данное исследование (результаты которого анонсированы в [1]) посвящено изучению топологии пространств вероятностных мер и находится на стьке двух дисциплин - бесконечномерной топологии и теории меры.

Интерес бесконечномерной топологии к исследованию пространств вероятностных мер объясняется тем фактом, что пространства мер, обладая дополнительной структурой выпуклости, являются идеальными, естественно возникающими в “природе" бесконечномерными объектами для приложения мощных методов бесконечномерной топологии. Среди важнейших достижений последнего времени в этой области нужно отметить характеризационные теоремы Х. Торуньчика для $Q-$ и $l^{2}$-многообразий [2], [3], а также созданную М. Бествиной и Е. Могильским теорию поглощающих множеств, позволяющую топологически характеризовать некоторые пространства, являющиеся универсальньми объектами в некоторых классах пространств [4]. Среди таких объектов нужно выделить построенные М. Бествиной и Е. Могильским подмножества $\Omega_{\alpha}$ и $\Lambda_{\alpha}$ гильбертова куба, являющиеся сильно универсальными пространствами для мультипликативного и аддитивного борелевских классов $\mathscr{M}_{\alpha}$ и $\mathscr{A}_{\alpha}$, где $\alpha$ - счетный ординал [4].

До недавнего времени, изучение пространств вероятностных мер проводилось в рамках категории компактов, где элементарной является задача топологической классификации: пространство вероятностных мер $P(X)$ компакта $X$ гомеоморфно либо конечномерному (если $X$ конечно), либо бесконечномерному кубу $[0,1]^{n}$, $0 \leqslant n \leqslant \infty$. За пределами компактов неясно уже, что именно считать пространством вероятностных мер на $X$. 
Существует как минимум четыре подхода к этому вопросу (дающие тот же результат для компактных $X$ ): пусть $X$ - тихоновское пространство. Рассмотрим следующие пространства мер (более подробно см. [5]-[8]):

(1) $P(\beta X)$ - пространство вероятностных мер на стоун-чеховской компактификации $\beta X$ пространства $X$, которое можно отождествить с пространством всех вероятностных конечно-аддитивных мер на $X$;

(2) $P_{\beta}(X)=\{\mu \in P(\beta X) \mid \operatorname{supp}(\mu) \subset X \subset \beta X\} \subset P(\beta X)-$ пространство вероятностных мер с компактными носителями на $X$;

(3) $P_{\tau}(X)=\left\{\mu \in P(\beta X) \mid 1=\mu^{*}(X)=\inf \{\mu(B) \mid B \supset X\right.$ - борелевское подмножество в $\beta X\}\} \subset P(\beta X)$ - пространство вероятностных $\tau$-гладких мер на $X$;

(4) $\widehat{P}(X)=\left\{\mu \in P(\beta X) \mid 1=\mu_{*}(X)=\sup \{\mu(B) \mid B \subset X-\right.$ борелевское подмножество в $\beta X\}\} \subset P(\beta X)$ - пространство вероятностных радоновских мер на $X$.

Основной объект, с которьм мы будем работать в этой статье - пространство $\widehat{P}(X)$ вероятностных радоновских мер на $X$. Выбор этот объясняется тем фактом, что, в отличие от конструкции пространства $P(\beta X)$, конструкция $\widehat{P}$ сохраняет метризуемость пространств (пространство $P(\beta X)$ метризуемо только в том случае, когда $X$ - метрический компакт). В то же время, пространство $\widehat{P}(X)$, в отличие от пространства $P_{\beta}(X)$, достаточно богато и содержит многие важные классы вероятностных мер (такие, например, как класс дискретных мер). Для некоторых пространств $X$ (например, для абсолютных борелевских) пространство $\widehat{P}(X)$ совпадает с пространством $P_{\tau}(X)$; более того, если $X$ - сепарабельное абсолютное борелевское множество, тогда пространство $\widehat{P}(X)$ содержит все счетно-аддитивные вероятностные меры на $X$.

Конструкция пространства $\widehat{P}(X)$ детально изучалась в [7], [8] с категорной и обшетопологической точек зрения. Там, в частности, доказано, что конструкция $\widehat{P}$ функториальна на категории тихоновских пространств. Более того, функтор $\widehat{P}$ сохраняет вложения, т.е. для пары пространств $(X, Y), Y \subset X$, пространство $\widehat{P}(Y)$ естественно вкладывается в $\widehat{P}(X)$, поэтому можно говорить о паре $(\widehat{P}(X), \widehat{P}(Y))$. Кроме того, в отличие от конструкции пространства $P_{\beta}(X)($ см. $[9],[10])$ конструкция $\widehat{P}$ чутко реагирует на изменение дескриптивных свойств $X$ : пространство $\widehat{P}(X)$ принадлежит мультипликативному классу $\mathscr{M}_{\alpha}$ абсолютных борелевских множеств, где $\alpha$ - счетный ординал, тогда и только тогда, когда $X \in \mathscr{M}_{\alpha}$ [11] либо $[7,2.30]$. Один из основных результатов этой статьи утверждает большее: для счетного ординала $\alpha$ и абсолютного борелевского пространства $X \in \mathscr{M}_{\alpha} \backslash \bigcup_{\xi<\alpha} \mathscr{M}_{\xi}$, пространство $\widehat{P}(X)$, в зависимости от бэровских свойств $X$, гомеоморфно либо $\Omega_{\alpha}$, либо $Q \backslash \Lambda_{\alpha}$, где $\Omega_{\alpha}$ и $\Lambda_{\alpha}$ - уже упоминавшиеся подмножества гильбертова куба $Q$, универсальные для борелевских классов $\mathscr{M}_{\alpha}$ и $\mathscr{A}_{\alpha}$.

Теперь немного об организации данной работы. Статья состоит из пяти параграфов. Первьй имеет подготовительньй характер - в нем собраны все необходимые в дальнейшем сведения.

Во втором параграфе, посвященном изучению топологии пространств вероятностных радоновских мер, мы получим полную топологическую классификацию пар вида $(\widehat{P}(X), \widehat{P}(Y))$, где $X$ - польское пространство и $Y$ - его всюду плотное борелевское подмножество (см. теоремы 2.7 и 2.8 ), откуда вытекает уже упоми- 
навшийся вьше классификационньй результат: для бесконечного сепарабельного абсолютного борелевского пространства $X$ пространство $\widehat{P}(X)$ гомеоморфно одному из пространств: $Q, \ell^{2}, \Omega_{\alpha}, Q \backslash \Lambda_{\alpha}, 2 \leqslant \alpha<\omega_{1}$. В частности, $\widehat{P}(X)$ гомеоморфно сепарабельному гильбертову пространству $\ell^{2}$ тогда и только тогда, когда $X$ - польское некомпактное пространство. Этот результат обобщается в теореме 2.11 на несепарабельный случай: пространство $\widehat{P}(X)$ гомеоморфно гильбертову пространству плотности $A$ тогда и только тогда, когда $X$ - полно-метризуемое (метризуемое полной метрикой) некомпактное пространство плотности $A$.

Третий параграф̆ посвящен изучению топологии пространства $P_{d}(X)$ вероятностных дискретных мер. Здесь $P_{d}(X)=\{\mu \in \widehat{P}(X) \mid \mu(C)=1$ для некоторого счетного подмножества $C \subset X\} \subset \widehat{P}(X)$. Мы получим полную топологическую классификацию пар вида $\left(\widehat{P}(K), P_{d}(X)\right)$, где $K$ - метрический компакт и $X \subset K$ - его всюду плотное $F_{\sigma \delta}$-подмножество (см. теорему 3.5$)$.

В четвертом параграфе исследуется подпространство $P_{c}(X) \subset \widehat{P}(X)$ вероятностных непрерывных мер (мера $\mu$ на $X$ называется непрерывной, если $\mu(\{x\})=0$ для любой точки $x \in X)$. В отличие от $\S 3$, где мы смогли идентифицировать топологию пространства $P_{d}(X)$ лиш для пространств $X$ маленькой борелевской сложности, здесь ситуация противоположная: мы получим топологическую классификацию пространств $P_{c}(X)$ лишш для достаточно сложных в борелевском отношении пространств $X$ (более конкретно, для борелевских пространств, не принадлежащих классу $\left.\left(\mathscr{M}_{2} \cap \mathscr{A}_{2}\right) \backslash \mathscr{M}_{1}\right)$, см. теоремы 4.5 и 4.8 .

Наконец, в пятом параграфе мы изучаем топологию пары $\left(\widehat{P}(X), P_{\omega}(Y)\right)$, где для $Y \subset X \quad P_{\omega}(Y)$ - подмножество $\widehat{P}(Y)$, состоящее из вероятностных мер с конечньми носителями. Т.Ф. Жураев [12] получил необходимые и достаточные условия на пару $(X, Y)$, при которых пара $\left(\widehat{P}(X), P_{\omega}(Y)\right)$ гомеоморфна паре $(Q, \sigma)$, где $\sigma=\left\{\left(t_{i}\right)_{i=1}^{\infty} \in s:\left|\left\{i \in \mathbb{N}: t_{i} \neq 0\right\}\right|<\infty\right\} \subset Q$. Несепарабельным аналогом пространства $\sigma$ является подмножество

$$
l_{f}^{2}(A)=\left\{\left(t_{a}\right)_{a \in A} \in \ell^{2}(A):\left|\left\{a \in A \mid t_{a} \neq 0\right\}\right|<\infty\right\} \subset \ell^{2}(A)
$$

гильбертова пространства плотности $A$. Основньп результатом $\S 5$ является теорема 5.1 , дающая необходимые и достаточные условия на пару $(X, Y)$, при которых пара $\left(\widehat{P}(X), P_{\omega}(Y)\right)$ гомеоморфна $\left(\ell^{2}(A), \ell_{f}^{2}(A)\right)$.

\section{§ 1. Предварительные сведения}

Все пространства, рассматриваемые в этой работе, предполагаются метризуемыми, а отображения - непрерывными. Через $\operatorname{cov}(X)$ обозначается множество всех открытых покрытий пространства $X$. Пусть $\mathscr{U} \in \operatorname{cov}(Y)$. Отображения $f, g: X \rightarrow Y$ называются $\mathscr{U}$-близкими (обозначается $(f, g) \prec \mathscr{U}$ ), если для любого $x \in X$ сушествует такое $U \in \mathscr{U}$, что $\{f(x), g(x)\} \subset U$. Напомним, что $\operatorname{St}(\mathscr{U})=\{\operatorname{St}(U, \mathscr{U}) \mid U \in \mathscr{U}\}$, где $\operatorname{St}(U, \mathscr{U})=\bigcup\{V \in \mathscr{U} \mid V \cap U \neq \varnothing\}$. Хорошо известно, что для каждого покрытия $\mathscr{U} \in \operatorname{cov}(Y)$ метрического пространства $(Y, d)$ существует такая положительная функция $\varepsilon: Y \rightarrow(0,1]$, что для каждой точки $y \in Y$ существует множество $U \in \mathscr{U}$ с $\left\{y^{\prime} \in Y \mid d\left(y, y^{\prime}\right)<\varepsilon(y)\right\} \subset U$. При этом функцию $\varepsilon$ можно выбрать липшицевой с коэффициентом 1 [4].

Польским называется любое сепарабельное полно-метризуемое пространство. Через $A(N) R$ обозначается класс абсолютных (окрестностных) ретрактов для 
класса метрических пространств. Хорошо известно, что каждое выпуклое подмножество локально выпуклого пространства является абсолютным ретрактом.

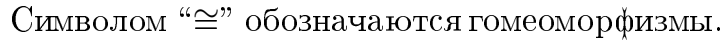

Замкнутое множество $A \subset X$ пространства $X$ называется $Z$-мно жеством в $X$, если любое отображение $f: Q \rightarrow X$ гильбертова кирпича $Q=[-1,1]^{\omega}$ равномерно аппроксимируется отображениями в $X \backslash A$. Подмножество $A \subset X$ пространства $X$ называется $\sigma Z$-множеством в $X$, если оно представляется в виде счетного объединения $Z$-множеств в $X$. Вложение $f: X \rightarrow Y$ называется $Z$-вложсением, если $f(Y)-Z$-множество в $X$. Подмножество $A \subset X$ называется гомотопически пренебрежимымм в $X$, если сушествует такая гомотопия $H: X \times[0,1] \rightarrow X$, что $H(x, 0)=x$ для всех $x \in X$ и $H(X \times(0,1]) \cap A=\varnothing$. Очевидно, что замкнутое гомотопически пренебрежимое множество является $Z$-множеством. Хорошо известно (см., например, [13]), что для всюду плотного выпуклого множества $Y \subset X$ метризуемого подмножества $X$ локально выпуклого пространства дополнение $X \backslash Y$ гомотопически пренебрежимо в $X$.

Напомним, что семейство $\mathscr{B}_{0}(X)$ всех борелевских подмножеств метризуемого пространства $X$ представляется в виде $\bigcup_{\alpha<\omega_{1}}\left(\mathscr{M}_{\alpha}(X) \cup \mathscr{A}_{\alpha}(X)\right)$, где $\mathscr{M}_{0}(X)$ $\left(\left(\mathscr{A}_{0}(X)\right)\right.$ - класс всех замкнутых (открытых) подмножеств пространства $X$. Для каждого счетного ординала $\alpha \mathscr{M}_{\alpha}(X)\left(\mathscr{A}_{\alpha}(X)\right)$ - семейство подмножеств $X$, представляемых в виде счетных пересечений (объединений) множеств из $\bigcup_{\xi<\alpha} \mathscr{A}_{\xi}(X)$ $\left(\bigcup_{\xi<\alpha} \mathscr{M}_{\xi}(X)\right)$.

Через $\mathscr{M}_{\alpha}\left(\mathscr{A}_{\alpha}\right)$ обозначается класс пространств, гомеоморфных подмножествам класса $\mathscr{M}_{\alpha}(Q)\left(\mathscr{A}_{\alpha}(Q)\right)$, где $Q=[-1,1]^{\omega}$ - гильбертов куб.

Семейство $\mathscr{C}$ топологических пространств называется:

(1) топологическим, если для любого гомеоморфизма $f: X \rightarrow Y$ из $X \in \mathscr{C}$ следует $Y \in \mathscr{C}$;

(2) замкнуто-аддитивным, если любое пространство $X=X_{1} \cup X_{2}$, являющееся объединением двух своих замкнутых подмножеств $X_{1}, X_{2} \in \mathscr{C}$, принадлежит классу $\mathscr{C}$;

(3) замкнуто-наследственныл, если для любого $X \in \mathscr{C}$ каждое замкнутое подмножество $Y \subset X$ принадлежит классу $\mathscr{C}$.

Хорошо известно, что классы $\mathscr{M}_{\alpha}$ и $\mathscr{A}_{\alpha}$ для $\alpha<\omega_{1}$, являются топологическими замкнуто-аддитивными и замкнуто-наследственными.

Пусть $X, K$ - пространства и $Y \subset X, L \subset K$. Пара $(X, Y)$ называется $(K, L)$-универсальной (соответственно, $(K, L)$-предуниверсальной), если сушествует замкнутоевложение (соответственно, совершенное отображение) $f: K \rightarrow X$ с $f^{-1}(Y)=L$. Пара $(X, Y)$ называется сильно $(K, L)$-универсальной, если для любого покрытия $\mathscr{U} \in \operatorname{cov}(X)$, любого замкнутого подмножества $B \subset K$ и отображения $f: K \rightarrow X$ такого, что $f \mid B: B \rightarrow X-Z$-вложение с $(f \mid B)^{-1}(Y)=L \cap B$, сушествует такое $Z$-вложение $h: K \rightarrow X$, что $(h, f) \prec \mathscr{U}, h|B=f| B$ и $h^{-1}(Y)=L$.

Для классов $\mathscr{K}, \mathscr{C}$ пространств через $(\mathscr{K}, \mathscr{C})$ обозначается класс всех пар $(K, C)$, где $\mathscr{K} \ni K \supset C \in \mathscr{C}$. Пара $(X, Y)$ называется сильно $(\mathscr{K}, \mathscr{C})$-универсальной (( $\mathscr{K}, \mathscr{C})$-(пред)универсальной), если она сильно $(K, L)$-универсальна $((K, L)$-(пред)универсальна) для любой пары $(K, L) \in(\mathscr{K}, \mathscr{C})$. 
Пара $(X, Y)$ назьвается $(\mathscr{K}, \mathscr{C})$-поглощающей, если

(1) $Y$ содержится в счетном объединении $\bigcup_{n=1}^{\infty} K_{n} Z$-множеств $K_{n} \subset X$, причем для любого $n \in \mathbb{N}\left(K_{n}, K_{n} \cap Y\right) \in(\mathscr{K}, \mathscr{C})$;

(2) пара $(X, Y)$ - сильно $(\mathscr{K}, \mathscr{C})$-универсальна.

Две пары $(X, Y)$ и $\left(X^{\prime}, Y^{\prime}\right)$ называются гомеоморфнылми, если $h(Y)=Y^{\prime}$ для некоторого гомеоморфизма $h: X \rightarrow X^{\prime}$.

Напомним, что $Q=[-1,1]^{\omega}$ - гильбертов куб, $s=(-1,1)^{\omega}$ - его псевдовнутренность и $\ell^{2}(A)=\left\{\left(x_{a}\right)_{a \in A} \mid x_{a} \in \mathbb{R}, \sum_{a \in A} x_{a}^{2}<\infty\right\}$ - стандартное гильбертово пространство плотности $A$. Если множество $A$ счетно, соответствующее сепарабельное гильбертово пространство обозначается через $\ell^{2}$. Хорошо известно, что пространство $\ell^{2}$ гомеоморфно псевдовнутренности $s$ гильбертова куба [14], [15].

Основным инструментом в нашем дальнейшем исследовании является следующая

1.1. ТеОрема $[16,1.7 .6]$. Пусть $\mathscr{K}, \mathscr{C}$ - топологические замкнуто-аддитивные замкнуто-наследственные классы пространств и пусть $F=Q$ либо $F=$ s. Любъе две $(\mathscr{K}, \mathscr{C})$-поглощающие парьи $(X, Y)$ u $\left(X^{\prime}, Y^{\prime}\right)$, где $X, X^{\prime}-$ топологические копии пространства $F$, гомеоморфны.

При применении теоремы 1.1 на практике, приходится проверять два перечисленных свойства, входящие в определение поглощающей пары, причем проверка сильной универсальности, как правило, технически сложна. На счастье, на данный момент, для пар вылуклых множеств получены глубокие результаты, позволяющие свести проверку сильной универсальности к доказательству предуниверсальности. Имеется ввиду следующая теорема, вытекающая из $[16,5.3 .5,5.2 .6$, 3.2.11 и 3.1.4].

1.2. ТЕОРемА. Пусть $L_{0}$ - локально-выпуклое линейное метрическое пространство, $L$ - его линейное подпространство и $K \subset L_{0}$ - такое выпуклое замкнутое подмножсество, что пересечение $K \cap L$ всюду плотно в $K$. Для любого счетного ординала $\alpha \geqslant 2$ пара $(K, K \cap L)$ сильно $\left(\mathscr{M}_{0}, \mathscr{M}_{\alpha}\right)$-универсальна тогда и только тогда, когда она $\left(\mathscr{M}_{0}, \mathscr{M}_{\alpha}\right)$-предуниверсальна.

Прилагая эту теорему к нашим задачам, мы, для метрического компакта $X$ и подмножества $Y \subset X$, будем полагать $K=P(X)$ и $K \cap L=\widehat{P}(Y)$, либо $K \cap L=P_{d}(Y)$, либо $K \cap L=P_{c}(Y)$ (пространства $\widehat{P}(Y), P_{d}(Y), P_{c}(Y)$, как легко видеть, являются пересечениями $P(X)$ с соответствующими линейньми подпространствами).

Пусть $X$ - топологическое пространство с $X^{\omega} \cong X$. Для подмножества $A \subset X$ и $* \in A$ положим $W(A, *)=\left\{\left(a_{i}\right)_{i=1}^{\infty} \in A^{\omega} \mid a_{i}=*\right.$ для почти всех $\left.i\right\} \subset X^{\omega}$. Для каждого счетного ординала $\alpha$ мы построим пары $\left(X, \Omega_{\alpha}(X)\right)$ и $\left(X, \Lambda_{\alpha}(X)\right)$, которые в случаях $X=Q$, либо $X=s$, являются соответственно $\left(\mathscr{M}_{0}(X), \mathscr{M}_{\alpha}(X)\right)$ и $\left(\mathscr{M}_{0}(X), \mathscr{A}_{\alpha}(X)\right)$-поглощающими. Положим $\left(X, \Lambda_{1}(X)\right) \cong\left(X^{\omega}, W(X, *)\right)$, где $* \in X$ и и $\left(X, \Omega_{1}(X)\right) \cong\left(X^{\omega}, W\left(X \backslash \Lambda_{1}(X), *\right)\right)$, где $* \in X \backslash \Lambda_{1}(X)$. Далее, предполагая, что для счетного ординала $\alpha$ пары $\left(X, \Omega_{\xi}(X)\right)$ и $\left(X, \Lambda_{\xi}(X)\right)$, где $\xi<\alpha$, уже построены, положим $\left(X, \Omega_{\alpha}(X)\right) \cong\left(X^{\omega}, \Lambda_{\beta}(X)^{\omega}\right)$, если $\alpha=\beta+1$ и $\left(X, \Omega_{\alpha}(X)\right) \cong\left(\prod_{\xi<\alpha} X^{\omega}, \prod_{\xi<\alpha} \Lambda_{\xi}(X)^{\omega}\right)$, если $\alpha$ - предельньй ординал; и 
$\left(X, \Lambda_{\alpha}(X)\right) \cong\left(X^{\omega}, W\left(X \backslash \Omega_{\alpha}(X), *\right)\right)$, где $* \in X \backslash \Omega_{\alpha}(X)$. Кроме того, положим $\Lambda_{0}(X)=\varnothing$.

Отметим, что для любого счетного ординала $\alpha$

$$
\left(s, \Lambda_{\alpha}(s)\right) \cong\left(Q \times s, \Lambda_{\alpha}(Q) \times s\right) \text { и }\left(s, \Omega_{\alpha}(s)\right) \cong\left(Q \times s, \Omega_{\alpha}(Q) \times s\right) .
$$

Это следует из построения пар $\left(s, \Omega_{\alpha}(s)\right)$ и $\left(s, \Lambda_{\alpha}(s)\right)$, а также из известного факта, утверждаюшего, что пары $\left(s^{\omega}, W(s, 0)\right)$ и $(Q \times s, \Sigma \times s)$ гомеоморфны (последнее является следствием теоремы 1 из [17]).

Еще несколько слов о конструкции $\widehat{P}(X)$ пространства вероятностных радоновских мер на $X$ (детали см. в [7], [8]). Напомним, что мерой на $X$ назьвается любая счетно-аддитивная функция $\mu: \mathscr{B}(X) \rightarrow[0, \infty)$, определенная на семействе $\mathscr{B}(X)$ всех борелевских подмножеств пространства $X$. При этом, мера $\mu$ называется $\boldsymbol{\theta} e-$ роятностной, если $\mu(X)=1$ и радоновской, если для любого борелевского множества $B \subset X$ и любого $\varepsilon>0$ существует компакт $K \subset B$ с $\mu(B \backslash K)<\varepsilon$.

Кроме внешнего описания (через стоун-чеховские компактификации), существует и внутреннее описание пространства $\widehat{P}(X)$ - это пространство всех вероятностных радоновских мер на $X$, наделенное топологией, предбазу которой составляют множества вида

$$
\left\{\mu \in \widehat{P}(X):\left|\int f d \mu-\int f d \mu_{0}\right|<\varepsilon\right\},
$$

где $\mu_{0} \in \widehat{P}(X)$ и $f: X \rightarrow \mathbb{R}$ - ограниченная непрерывная функция на $X$.

Если $(X, d)$ - ограниченное метрическое пространство, тогда пространство $\widehat{P}(X)$ метризуемо, причем метрика $\widehat{d}$ на $\widehat{P}(X)$ может быть выписана в явном виде:

$$
\widehat{d}\left(\mu_{1}, \mu_{2}\right)=\inf \left\{\lambda(d) \mid \lambda \in \widehat{P}(X \times X), \widehat{P}\left(\mathrm{pr}_{i}\right)(\lambda)=\mu_{i}, i=1,2\right\},
$$

где $\operatorname{pr}_{i}: X \times X \rightarrow X$ - соответствуюшие проекции. При этом, если $d$-полная метрика на $X$, тогда $\widehat{d}$ - полная метрика на $\widehat{P}(X)$. Мы будем использовать следуюшее свойство метрики $\widehat{d}$ (доказанное в $[8, \S 4]): \widehat{d}((1-t) \mu+t \eta, \mu) \leqslant t \widehat{d}(\mu, \eta)$ для любых $t \in[0,1]$ и мер $\mu, \eta \in \widehat{P}(X)$.

Если $Y$ - борелевское подмножество пространства $X$, тогда пространство $\widehat{P}(Y)$ может быть отождествлено с подмножеством $\{\mu \in \widehat{P}(X) \mid \mu(Y)=1\}$ в $\widehat{P}(X)$. Как мы уже упоминали, для любого счетного ординала $\alpha Y \in \mathscr{M}_{\alpha}(X)$ тогда и только тогда, когда $\widehat{P}(Y) \in \mathscr{M}_{\alpha}(\widehat{P}(X))[11]$. В частности, если $F$ - замкнутое множество в $X$, тогда $\widehat{P}(F)$ замкнуто в $\widehat{P}(X)$. Более того, для любого $a \in \mathbb{R}$ множество $\{\mu \in \widehat{P}(X) \mid \mu(F) \geqslant a\}$ тоже замкнуто в $\widehat{P}(X)($ см. $[7,1.19])$.

\section{§2. Топология пространств $\widehat{P}(X)$}

В этом параграфе мы получим полную топологическую классификацию пар вида $(\widehat{P}(X), \widehat{P}(Y))$, где $X$ - польское пространство и $Y \subset X$ - его всюду плотное борелевское подмножество.

Если $X$ - метризуемое пространство, то $\widehat{P}(X)$ является метризуемым выпукльм подмножеством локально-вьпуклого пространства $($ см. $[7, \S 2])$. Отсюда и из обобшенной теоремы Титце [14] вытекает 
2.1. ПРЕДЛОЖЕНИЕ. Для любого метризуемого пространства $X$ пространство $\widehat{P}(X)$ является абсолютным ретрактом для класса метрических пространств.

Напомним, что $X$ называется пространством первой категории, если $X$ представляется в виде счетного объединения нигде не плотных подмножеств. Если в $X$ сушествует непустое открытое подмножество, являюшееся пространством первой категории, тогда $X$ назьвается пространством әде-то первой категории; $X$ называется бәровским пространством, если для любой последовательности $\left\{U_{n}\right\}$ открытых всюду плотных подмножеств $X$, пересечение $\bigcap_{n=1}^{\infty} U_{n}$ всюду плотно в $X$. Хорошо известно, что класс пространств где-то первой категории совпадает с классом пространств, не являющихся бэровскими.

2.2. ПРЕДЛОЖЕНИЕ. Пусть $Y \subset X$ - пара метрических пространств. Если пространство $Y$ не является бэровским, тогда $\widehat{P}(Y)$ содержится в $\sigma Z$-мнохестве в $\widehat{P}(X)$.

ДоказАТЕЛьство. Пусть $U \subset Y$ - непустое открытое множество в $Y$, являющееся пространством первой категории. Положим $F=\mathrm{cl}_{X}(Y \backslash U)$. Поскольку $U$ - множество первой категории, $U \subset \bigcup_{n=1}^{\infty} F_{n}$, где $F_{n} \subset F_{n+1} \subset X, n \in \mathbb{N},-$ замкнутые нигде не плотные множества в $X$. Очевидно, что $Y \subset F \cup\left(\bigcup_{n=1}^{\infty} F_{n}\right)$. Тогда $\widehat{P}(Y)=\widehat{P}(F) \cup\left(\bigcup_{n=1}^{\infty} A_{n}\right)$, где $A_{n}=\left\{\mu \in \widehat{P}(X) \mid \mu\left(F_{n}\right) \geqslant 2^{-n}\right\}, n \geqslant 1$. Поскольку $F \neq X$, то $\widehat{P}(F)-Z$-множество в $\widehat{P}(X)$. Действительно, множество $\widehat{P}(F)$ замкнуто в $\widehat{P}(X)$. Кроме того, $\widehat{P}(X) \backslash \widehat{P}(F)$ - выпуклое всюду плотное подмножество в $A R$-пространстве $\widehat{P}(X)$. Отсюда следует, что замкнутое множество $\widehat{P}(F)$ - гомотопически пренебрежимо в $\widehat{P}(X)$, а значит, является $Z$-множеством в $\widehat{P}(X)$. Аналогично, множества $A_{n}, n \in \mathbb{N}$, являются $Z$-множествами в $\widehat{P}(X)$ (поскольку $F_{n}$ - нигде не плотное замкнутое множество, то $A_{n}$ - замкнутое подмножество $\widehat{P}(X)$, причем его дополнение $\widehat{P}(X) \backslash A_{n}$ - выпуклое всюду плотное множество в $\widehat{P}(X))$. Таким образом, множество $\widehat{P}(Y)$ содержится в $\sigma Z$-множестве $\widehat{P}(F) \cup\left(\bigcup_{n=1}^{\infty} A_{n}\right) \subset \widehat{P}(X)$.

2.3. ПРЕДЛОЖЕНИЕ. Пусть $Y$ - всюду плотное борелевское подмножество метрического пространства $X$. Если пространство $Y$ - бәровское, тогда $\widehat{P}(X) \backslash \widehat{P}(Y)$ содерәится в $\sigma Z$-множестве в $\widehat{P}(X)$.

ДоКАЗАТЕЛЬСтво. Поскольку $Y$ - борелевское подмножество в $X$, то $Y=G \cup$ $F$, где $G-G$-подмножество и $F-$ множество первой категории в $X[18, \S 11]$. Тогда $\widehat{P}(X) \backslash \widehat{P}(Y) \subset \widehat{P}(X) \backslash \widehat{P}(G)$. Поскольку пространство $Y$ - бэровское, то $G$ - всюду плотное множество в $X$. Тогда $X \backslash G=\bigcup_{n=1}^{\infty} F_{n}$, где $F_{n} \subset F_{n+1}$, $n \in \mathbb{N},-$ замкнутые нигде не плотные подмножества в $X$. Следовательно, $\widehat{P}(X) \backslash$ $\widehat{P}(G)=\bigcup_{n=1}^{\infty} A_{n}$, где $A_{n}=\left\{\mu \in \widehat{P}(X) \mid \mu\left(F_{n}\right) \geqslant 2^{-n}\right\}, n \in \mathbb{N}$. Поскольку $F_{n}, n \in \mathbb{N}$, - замкнутые нигде не плотные подмножества $X$, то $A_{n}-Z$-множество в $\widehat{P}(X)$ для каждого $n \in \mathbb{N}$. Таким образом, $\widehat{P}(X) \backslash \widehat{P}(Y) \subset \widehat{P}(X) \backslash \widehat{P}(G) \subset \bigcup_{n=1}^{\infty} A_{n}$ содержится в $\sigma Z$-подмножестве $\widehat{P}(X)$.

2.4. ТЕОремА. Пусть $X$ - бесконечный метрический компакт $и \neq X$ его всюду плотное $G_{\delta}$-подмножество. Тогда пара $(P(X), \widehat{P}(Y))$ гомеоморфна nape $(Q, s)$. 
ДокАЗАТЕЛЬСтво. Достаточно показать, что пара $(P(X), P(X) \backslash \widehat{P}(Y))$ гомеоморфна паре $(Q, \Sigma)$. Поскольку $X$ - бесконечньй компакт, $P(X)$ бесконечномерный выпукльй компакт в локально выпуклом пространстве, который, в силу теоремы Келлера-Кли $[15,8.2 .4]$, гомеоморфен гильбертову кирпичу $Q$. Отметим, что множество $P(X) \backslash \widehat{P}(Y)$ - выпукло и всюду плотно в $P(X)$. Поскольку $\widehat{P}(Y)$ - выпуклое всюду плотное множество в $P(X)$, то множество $P(X) \backslash \widehat{P}(Y)$ - гомотопически пренебрежимо в $P(X)$. Следовательно, каждый компакт в $P(X) \backslash \widehat{P}(Y)$ является $Z$-множеством в $P(X)$. Далее, пространство $P(X) \backslash \widehat{P}(Y)$ содержит бесконечномерный выпукльй компакт $K=\left\{\mu \in P(X) \mid \mu(\{x\}) \geqslant \frac{1}{2}\right\}$, где $x \in X \backslash Y$. Согласно предложению 3.4 [19] множество $P(X) \backslash \widehat{P}(Y)$ содержит скелетоид для компактов. Поскольку $Y-$ $G_{\delta}$-множество в $X$, то $\widehat{P}(Y)-G_{\delta}$-множество в $P(X)$ (см. [11] либо $\left.[7,2.30]\right)$. Отсюда $P(X) \backslash \widehat{P}(Y)-\sigma$-компактное множество, а, следовательно, и $\sigma Z$-множество в $P(X)$. По $[15, \S 6.5]$ пара $(P(X), P(X) \backslash \widehat{P}(Y))$ гомеоморфна паре $(Q, s)$.

2.5. ПРЕДЛОЖЕНИЕ. Пусть $1 \leqslant \alpha<\omega_{1}$ - счетный ординал и $Y \notin$ $\bigcup_{\xi<\alpha} \mathscr{M}_{\xi}(X)$ - всюду плотное борелевское подмножество метрического компакта $X$. Тогда пара $(\widehat{P}(X), \widehat{P}(Y))$ сильно $\left(\mathscr{M}_{0}, \mathscr{M}_{\alpha}\right)$-универсальна.

Для доказательства нам понадобятся некоторые вспомогательные леммы.

Через $C=\{0,1\}^{\omega}$ обозначим канторов куб. По аналогии с парами $\left(C, \Lambda_{\alpha}(C)\right)$, $\left(C, \Omega_{\alpha}(C)\right)$, для каждого счетного ординала $\alpha$ мы построим пары $\left(C, A_{\alpha}(C)\right)$, $\left(C, M_{\alpha}(C)\right)$. Положим $\left(C, M_{1}(C)\right) \cong\left(C^{\omega},\left(C \backslash \Lambda_{1}(C)\right)^{\omega}\right)$ и $\left(C, A_{1}(C)\right)=$ $\left(C, C \backslash M_{1}(C)\right)$. Предположив, что для счетного ординала $\alpha$ пары $\left(C, M_{\xi}(C)\right)$ и $\left(C, A_{\xi}(C)\right)$, где $\xi<\alpha$, уже построены, положим $\left(C, M_{\alpha}(C)\right) \cong\left(C^{\omega}, A_{\beta}(C)^{\omega}\right)$, если $\alpha=\beta+1 ;$ и $\left(C, M_{\alpha}(C)\right) \cong\left(\prod_{\xi<\alpha} C^{\omega}, \prod_{\xi<\alpha} A_{\xi}(C)^{\omega}\right)$, если $\alpha-$ предельный ординал; и $\left(C, A_{\alpha}(C)\right)=\left(C, C \backslash M_{\alpha}(C)\right)$. Легко видеть, что $A_{\alpha}(C) \in \mathscr{A}_{\alpha}(C)$ и $M_{\alpha}(C) \in \mathscr{M}_{\alpha}(C)$.

2.6. Лемма. Для каждого счетного ординала $\alpha \geqslant 1$ пара $\left(P(C), \widehat{P}\left(A_{\alpha}(C)\right)\right)$ $\left(\mathscr{M}_{0}, \mathscr{M}_{\alpha+1}\right)$-предуниверсальна.

ДоказАтельство. Покажем сначала, что пара $\left(P(C), \widehat{P}\left(A_{1}(C)\right)\right)\left(\mathscr{M}_{0}, \mathscr{A}_{1}\right)$ предуниверсальна. По теореме 2.4 пара $\left(P(C), \widehat{P}\left(M_{1}(C)\right)\right)$ гомеоморфна паpe $(Q, s)$. Пусть $h:(Q, s) \rightarrow\left(P(C), \widehat{P}\left(M_{1}(C)\right)\right)$ - соответствующий гомеоморфизм пар. Положим $H: Q \rightarrow P\left(C^{\omega}\right)$ - отображение, определенное формулой $H(q)=h(q) \otimes h(q) \otimes \cdots-$ бесконечное тензорное произведение мер $h(q) \in P(C)$ для $q \in Q$ (см. $[20, \S 2.6]$, либо $[7, \S 2])$. При этом $H(s) \subset \widehat{P}\left(M_{1}(C)^{\omega}\right)$ и $H(\Sigma) \subset \widehat{P}\left(C^{\omega} \backslash\left(M_{1}(C)\right)^{\omega}\right)$, где $\Sigma=Q \backslash s$. Теперь пусть $f:\left(C^{\omega}, M_{1}(C)^{\omega}\right) \rightarrow\left(C, M_{1}(C)\right)$ - гомеоморфизм пар. Тогда отображение $\xi=P(f) \circ H: Q \rightarrow P(C)$ обладает следуюшими свойствами: $\xi(s) \subset \widehat{P}\left(M_{1}(C)\right)$ и $\xi(\Sigma) \subset \widehat{P}\left(C \backslash M_{1}(C)\right)$. Следовательно, $\xi^{-1}\left(\widehat{P}\left(A_{1}(C)\right)\right)=\Sigma$. Поскольку пара $(Q, \Sigma)$ сильно $\left(\mathscr{M}_{0}, \mathscr{A}_{1}\right)$-универсальна $[15, \S 6.5]$, то она $\left(\mathscr{M}_{0}, \mathscr{A}_{1}\right)$-предуниверсальна. Следовательно, и пара $\left(P(C), \widehat{P}\left(A_{1}(C)\right)\right) \quad\left(\mathscr{M}_{0}, \mathscr{A}_{1}\right)$-предуниверсальна. Покажем, что она $\left(\mathscr{M}_{0}, \mathscr{M}_{2}\right)$-предуниверсальна. Пусть $K$-компакт и $K \supset L \in \mathscr{M}_{2}$. Тогда $L=\bigcap_{n=1}^{\infty} L_{n}$, где $K \supset L_{n} \in \mathscr{A}_{1}, n \in \mathbb{N}$. Поскольку пара $\left(P(C), \widehat{P}\left(A_{1}(C)\right)\right)$ $\left(\mathscr{M}_{0}, \mathscr{A}_{1}\right)$-предуниверсальна, то для каждого $n \in \mathbb{N}$ сушествует такое непрерывное отображение $\xi_{n}: K \rightarrow P(C)$, что $\xi_{n}^{-1}\left(\widehat{P}\left(A_{1}(C)\right)\right)=L_{n}$. Тогда отображение 
$\xi: K \rightarrow P(C)$, определенное формулой $\xi(q)=\sum_{n=1}^{\infty} 2^{-n} \xi_{n}(g), q \in K$, обладает свойством:

$$
\xi^{-1}\left(\widehat{P}\left(A_{1}(C)\right)\right)=\bigcap_{n=1}^{\infty} L_{n}=L .
$$

То есть, пара $\left(P(C), \widehat{P}\left(A_{1}(C)\right)\right)\left(\mathscr{M}_{0}, \mathscr{M}_{2}\right)$-предуниверсальна.

Пусть $\alpha>1$ - счетный ординал. Предположим, что для всех ординалов $\xi<\alpha$ мы уже доказали, что пара $\left(P(C), \widehat{P}\left(A_{\xi}(C)\right)\right)\left(\mathscr{M}_{0}, \mathscr{M}_{\xi+1}\right)$-предуниверсальна. Докажем сначала, что пара $\left(P(C), \widehat{P}\left(A_{\alpha}(C)\right)\right)\left(\mathscr{M}_{0}, \mathscr{A}_{\alpha}\right)$-предуниверсальна.

Предположим, что $\alpha=\beta+1$ - непредельный ординал. Пусть $K$ - компакт и $K \supset L \in \mathscr{A}_{\alpha}$. Тогда $K \backslash L \in \mathscr{M}_{\alpha}$. Согласно предположению трансфинитной индукции, пара $\left(P(C), \widehat{P}\left(A_{\beta}(C)\right)\right)$ ( $\left.\mathscr{M}_{0}, \mathscr{M}_{\alpha}\right)$-предуниверсальна. Следовательно, существует такое непрерывное отображение $\xi: K \rightarrow P(C)$, что $\xi^{-1}\left(\widehat{P}\left(A_{\beta}(C)\right)\right)=$ $K \backslash L$. Определим отображение $H: K \rightarrow P\left(C^{\omega}\right)$ формулой $H(x)=\xi(x) \otimes \xi(x) \otimes \cdots$, $x \in K$. Легко видеть, что $H(K \backslash L) \subset \widehat{P}\left(A_{\beta}(C)^{\omega}\right)$ и $H(L) \subset \widehat{P}\left(C^{\omega} \backslash\left(A_{\beta}(C)\right)^{\omega}\right)$. Пусть $h: C^{\omega} \rightarrow C$-гомеоморфизм, для которого $h\left(C^{\omega} \backslash A_{\beta}(C)^{\omega}\right)=A_{\alpha}(C)$. Тогда отображение $f=P(h) \circ H: K \rightarrow P(C)$ обладает следующим свойством:

$$
f^{-1}\left(\widehat{P}\left(A_{\alpha}(C)\right)\right)=L
$$

Таким образом, пара $\left(P(C), \widehat{P}\left(A_{\alpha}(C)\right)\right)\left(\mathscr{M}_{0}, \mathscr{A}_{\alpha}\right)$-предуниверсальна.

Теперь пусть $\alpha$ - предельный ординал, $K$ - компакт и $K \supset L \in \mathscr{A}_{\alpha}$. Тогда $K \backslash L \in \mathscr{M}_{\alpha}$ и, следовательно,

$$
K \backslash L=\bigcap_{n=1}^{\infty} N_{n}
$$

где

$$
N_{n} \in \bigcup_{\xi<\alpha} \mathscr{M}_{\xi}=\bigcup_{\xi<\alpha} \mathscr{A}_{\xi}
$$

для каждого $n \in \mathbb{N}$. Поскольку

$$
\left(C, M_{\alpha}(C)\right) \cong\left(\prod_{\xi<\alpha} C^{\omega}, \prod_{\xi<\alpha} A_{\xi}(C)^{\omega}\right)
$$

и для каждого $\xi<\alpha$ пара $\left(P(C), \widehat{P}\left(A_{\xi}(C)\right)\right) \quad\left(\mathscr{M}_{0}, \mathscr{M}_{\xi+1}\right)$-предуниверсальна, то для каждого $n \in \mathbb{N}$ существует такое отображение $\xi_{n}: K \rightarrow P(C)$, что $\xi_{n}^{-1}\left(\widehat{P}\left(M_{\alpha}(C)\right)\right)=N_{n}$. Заменяя, если необходимо, отображение $\xi_{n}$ его счетной тензорной степенью и используя гомеоморфизм $\left(C, M_{\alpha}(C)\right) \cong\left(C^{\omega}, M_{\alpha}(C)^{\omega}\right)$, можно добиться чтобы $\xi_{n}\left(N_{n}\right) \subset \widehat{P}\left(M_{\alpha}(X)\right)$ и $\xi_{n}\left(K \backslash N_{n}\right) \subset \widehat{P}\left(C \backslash M_{\alpha}(C)\right)$. Рассмотрим теперь поточечное тензорное произведение

$$
H=\bigotimes_{n=1}^{\infty} \xi_{n}: K \rightarrow P\left(C^{\omega}\right)
$$


Нетрудно убедиться, что $H(K \backslash L) \subset \widehat{P}\left(M_{\alpha}(C)^{\omega}\right)$ и $H(L) \subset \widehat{P}\left(C^{\omega} \backslash M_{\alpha}(C)^{\omega}\right)$. Пусть $h:\left(C^{\omega}, M_{\alpha}(C)^{\omega}\right) \rightarrow\left(C, M_{\alpha}(C)\right)$ - гомеоморфизм пар. Тогда отображение $f=P(h) \circ H: K \rightarrow P(C)$ обладает свойством:

$$
f^{-1}\left(\widehat{P}\left(C \backslash M_{\alpha}(C)\right)\right)=f^{-1}\left(\widehat{P}\left(A_{\alpha}(C)\right)\right)=L .
$$

Таким образом, пара $\left(P(C), \widehat{P}\left(A_{\alpha}(C)\right)\right)\left(\mathscr{M}_{0}, \mathscr{A}_{\alpha}\right)$-предуниверсальна.

Покажем, что пара $\left(P(C), \widehat{P}\left(A_{\alpha}(C)\right)\right)\left(\mathscr{M}_{0}, \mathscr{M}_{\alpha+1}\right)$-предуниверсальна. Пусть $K$ - компакт и $K \supset L \in \mathscr{M}_{\alpha+1}$. Тогда $L=\bigcap_{n=1}^{\infty} L_{n}$, где $K \supset L_{n} \in \mathscr{A}_{\alpha}$, $n \in \mathbb{N}$. Поскольку пара $\left(P(C), \widehat{P}\left(A_{\alpha}(C)\right)\right)\left(\mathscr{M}_{0}, \mathscr{A}_{\alpha}\right)$-предуниверсальна, для каждого $n \in \mathbb{N}$ существует такое непрерывное отображение $\xi_{n}: K \rightarrow P(C)$, что $\xi_{n}^{-1}\left(\widehat{P}\left(A_{\alpha}(C)\right)\right)=L_{n}$. Тогда отображение $\xi: K \rightarrow P(C)$, определенное формулой $\xi(x)=\sum_{n=1}^{\infty} 2^{-n} \xi_{n}(x), x \in K$, обладает свойством

$$
\xi^{-1}\left(\widehat{P}\left(A_{\alpha}(C)\right)\right)=L .
$$

Таким образом, пара $\left(P(C), \widehat{P}\left(A_{\alpha}(C)\right)\right)\left(\mathscr{M}_{0}, \mathscr{M}_{\alpha+1}\right)$-предуниверсальна.

ДОКАЗАТЕЛЬСТВО ПРЕДЛОЖЕНИЯ 2.5. Пусть $1 \leqslant \alpha<\omega_{1}-$ счетньй ординал и $Y \notin \bigcup_{\xi<\alpha} \mathscr{M}_{\xi}(X)$ - всюду плотное борелевское подмножество компакта $X$. Если $Y \in \mathscr{M}_{1} \backslash \mathscr{M}_{0}$, то из теоремы 2.4 следует, что пара $(P(X), \widehat{P}(Y))$ гомеоморфна паре $(Q, s)$ и, следовательно, является сильно $\left(\mathscr{M}_{0}, \mathscr{M}_{1}\right)$-универсальной. Поэтому далее мы предполагаем, что $Y \notin \mathscr{M}_{1}$.

Далее, рассмотрим два случая:

(1) $\alpha=\beta+1$ - непредельньй ординал;

(2) $\alpha$ - предельньй ординал.

1) Пусть $\alpha=\beta+1$. Тогда $Y \notin \mathscr{M}_{\beta}$. Согласно [21, 22.13], сушествует такое отображение $\xi: C \rightarrow X$, что $\xi^{-1}(Y)=A_{\beta}(C)$. Поскольку функтор $\widehat{P}$ сохраняет прообразы (см. [7, 2.14]), то для отображения $\widehat{P}(\xi): \widehat{P}(C) \rightarrow \widehat{P}(X)$ выполняется равенство $\widehat{P}(\xi)^{-1}(\widehat{P}(Y))=\widehat{P}\left(A_{\beta}(C)\right)$. По лемме 2.6 пара $\left(P(C), \widehat{P}\left(A_{\beta}(C)\right)\left(\mathscr{M}_{0}, \mathscr{M}_{\alpha}\right)\right.$ предуниверсальна. Следовательно, пара $(P(X), \widehat{P}(Y))$ тоже $\left(\mathscr{M}_{0}, \mathscr{M}_{\alpha}\right)$-предуниверсальна. По теореме 1.2 пара $(P(X), \widehat{P}(Y))$ сильно $\left(\mathscr{M}_{0}, \mathscr{M}_{\alpha}\right)$-универсальна.

Теперь пусть $\alpha$ - предельный ординал и пусть $\left\{\alpha_{n}\right\}$ - монотонная последовательность ординалов $<\alpha$, сходящаяся к $\alpha$.

Пусть $K$-компакт и $L \subset K \in \mathscr{M}_{\alpha}$. Тогда множество $L$ представимо в виде пересечения $L=\bigcap_{n=1}^{\infty} L_{n}$, где $L_{n} \subset K$ - подмножества класса $\mathscr{M}_{\alpha_{n}}$. Из доказанного вьше следует, что для каждого $n \in \mathbb{N}$ пара $(P(X), \widehat{P}(Y))\left(\mathscr{M}_{0}, \mathscr{M}_{\alpha_{n}}\right)$-предуниверсальна, откуда вытекает существование непрерьвного отображения $\xi_{n}: K \rightarrow$ $P(X)$ со свойством $\xi_{n}^{-1}(\widehat{P}(Y))=L_{n}$. Пусть $\xi: K \rightarrow P(X)$ - отображение, определенное формулой $\xi(x)=\sum_{n=1}^{\infty} 2^{-n} \xi_{n}(x)$. Легко видеть, что $\xi^{-1}(\widehat{P}(Y))=L$, т.е. пара $(P(X), \widehat{P}(Y))\left(\mathscr{M}_{0}, \mathscr{M}_{\alpha}\right)$-предуниверсальна.

По теореме 1.2 , пара $(P(X), \widehat{P}(Y))$ сильно $\left(\mathscr{M}_{0}, \mathscr{M}_{\alpha}\right)$-универсальна.

2.7. Теорема. Пусть $\alpha \geqslant 1$ - счетный ординал, $X$ - метрический компакт и $Y \in \mathscr{M}_{\alpha} \backslash \bigcup_{\xi<\alpha} \mathscr{M}_{\xi}$ - всюду плотное подмножсество в $X$. Если $Y$ - бәровское пространство, тогда пара $(P(X), \widehat{P}(Y))$ гомеоморфна паре $\left(Q, Q \backslash \Lambda_{\alpha}(Q)\right) ;$ в противном случае, пара $(P(X), \widehat{P}(Y))$ гомеоморфна паре $\left(Q, \Omega_{\alpha}(Q)\right)$. 
ДокАЗАТЕЛЬСТво. Поскольку $Y$ - всюду плотное собственное подмножество $X$, то $X$ - бесконечный компакт. Следовательно, пространство $P(X)$ гомеоморфно гильбертову кубу $Q$. Из [11] следует, что $\widehat{P}(Y) \in \mathscr{M}_{\alpha}(P(X))$.

При $\alpha=1$, утверждение теоремы 2.7 следует из теоремы 2.4 , поскольку пара $(Q, s)$ гомеоморфна паре $\left(Q, Q \backslash \Lambda_{1}(Q)\right)$.

Предположим, что пространство $Y$ не является бэровским. Согласно предложению $2.2, \widehat{P}(Y)$ содержится в $\sigma Z$-множестве в $\widehat{P}(X)$. Кроме того, из предложения 2.5 вытекает, что пара $(P(X), \widehat{P}(Y))$ сильно $\left(\mathscr{M}_{0}, \mathscr{M}_{\alpha}\right)$-универсальна. Таким образом, пара $(P(X), \widehat{P}(Y)) \quad\left(\mathscr{M}_{0}, \mathscr{M}_{\alpha}\right)$-поглощающая и, по теореме 1.1 , гомеоморфна стандартной $\left(\mathscr{M}_{0}, \mathscr{M}_{\alpha}\right)$-поглошаюшей паре $\left(Q, \Omega_{\alpha}(Q)\right)$.

Если пространство $Y$ - бэровское, то из предложения 2.3 следует, что множество $P(X) \backslash \widehat{P}(Y)$ содержится в $\sigma Z$-множестве в $P(X)$. Кроме того, поскольку $\widehat{P}(Y) \in \mathscr{M}_{\alpha}$, то $P(X) \backslash \widehat{P}(Y) \in \mathscr{A}_{\alpha}$. Далее, поскольку пара $(P(X), \widehat{P}(Y))$ сильно $\left(\mathscr{M}_{0}, \mathscr{M}_{\alpha}\right)$-универсальна, то пара $(P(X), P(X) \backslash \widehat{P}(Y))$ сильно $\left(\mathscr{M}_{0}, \mathscr{A}_{\alpha}\right)$-универсальна. Таким образом, пара $(P(X), P(X) \backslash \widehat{P}(Y))\left(\mathscr{M}_{0}, \mathscr{A}_{\alpha}\right)$-поглощающая и по теореме 1.1 гомеоморфна стандартной $\left(\mathscr{M}_{0}, \mathscr{A}_{\alpha}\right)$-поглошаюшей паре $\left(Q, \Lambda_{\alpha}(Q)\right)$. Следовательно, $(P(X), \widehat{P}(Y)) \cong\left(Q, Q \backslash \Lambda_{\alpha}(Q)\right)$.

2.8. ТеОРема. Пусть $\alpha \geqslant 1$ - счетный ординал, $X$ - польское некомпактное пространство и $Y \in \mathscr{M}_{\alpha} \backslash \cup_{\xi<\alpha} \mathscr{M}_{\xi}$ - его всюду плотное собственное подмножество. Пара $(\widehat{P}(X), \widehat{P}(Y))$ гомеоморфна

(1) паре $\left(s, s \backslash \Lambda_{\alpha}(s)\right)$, если $Y$ - бәровское пространство;

(2) паре $\left(s, \Omega_{\alpha}(s)\right)$ в противном случае.

ДокАЗАтЕльство. Рассуждая аналогично, как при доказательстве 2.7, мы видим, что доказательство теоремы 2.8 сводится к проверке сильной $\left(\mathscr{M}_{1}, \mathscr{M}_{\alpha}\right)$ универсальности пары $(\widehat{P}(X), \widehat{P}(Y))$. Мы будем использовать следующий факт, вытекающий из результатов [22] (см. также [16, 5.3.2]).

2.9. ЛЕмма. Пусть $X$ - польское пространство, $Y$ - его всюду плотное подмножество, и $\alpha \geqslant 1$ - счетный ординал. Если для некоторого замкнутого подмножества $A \subset X$ с бесконечныцм дополнением пара $(\widehat{P}(A), \widehat{P}(A \cap Y))$ $\left(\mathscr{M}_{1}, \mathscr{M}_{\alpha}\right)$-универсальна, тогда пара $(\widehat{P}(X), \widehat{P}(Y))$ сильно $\left(\mathscr{M}_{1}, \mathscr{M}_{\alpha}\right)$-универсальна.

Нам понадобится также следуюшее тривиальное

2.10. ЗАмечанИЕ. Если пара $(A, B)$ ( $\left.\mathscr{M}_{0}, \mathscr{M}_{\alpha}\right)$-универсальна, тогда пара $(A \times s, B \times s)\left(\mathscr{M}_{1}, \mathscr{M}_{\alpha}\right)$-универсальна.

Итак, для доказательства 2.8 , нам достаточно найти замкнутое подмножество $A \subset X$ с бесконечным дополнением, для которого пара $(\widehat{P}(A), \widehat{P}(A \cap Y))$ $\left(\mathscr{M}_{1}, \mathscr{M}_{\alpha}\right)$-универсальна. Как и в 2.6 , мы рассматриваем два случая.

I) $\alpha=\beta+1$ - непредельный ординал. Поскольку $Y \notin \mathscr{M}_{\beta}$, то существует такой компакт $K \subset X$, что $K \cap Y \notin \mathscr{M}_{\beta}$ [21, 22.13 и 24.20]. Согласно 2.5 пара $(P(K), P(K \cap Y))\left(\mathscr{M}_{0}, \mathscr{M}_{\alpha}\right)$-универсальна.

Поскольку пространство $X$ некомпактно и $Y$ всюду плотно в $X$, то $X$ содержит замкнутое дискретное подмножество $N \subset Y$. Без ограничения обшности, 
$K \cap N=\varnothing$ и $X \backslash(K \cup N)$ бесконечно. По теореме 2.4 пространство $\widehat{P}(N) \subset \widehat{P}(Y)$ гомеоморфно $s$. Рассмотрим подмножество $\frac{1}{2} P(K)+\frac{1}{2} \widehat{P}(N) \subset \widehat{P}(K \cup N)$ и отметим, что оно замкнуто; более того, пара

$$
\begin{aligned}
\left(\frac{1}{2} P(K)+\frac{1}{2} \widehat{P}(N),\left(\frac{1}{2} P(K)+\right.\right. & \left.\left.\frac{1}{2} \widehat{P}(N)\right) \cap \widehat{P}(Y)\right) \\
& =\left(\frac{1}{2} P(K)+\frac{1}{2} \widehat{P}(N), \frac{1}{2} P(K \cap Y)+\frac{1}{2} \widehat{P}(N)\right)
\end{aligned}
$$

гомеоморфна паре

$$
(P(K) \times \widehat{P}(N), \widehat{P}(K \cap Y) \times \widehat{P}(N)),
$$

которая в силу $2.10\left(\mathscr{M}_{1}, \mathscr{M}_{\alpha}\right)$-универсальна. Поскольку множество $\frac{1}{2} P(K)+$ $\frac{1}{2} \widehat{P}(N)$ замкнуто в $\widehat{P}(K \cup N)$, отсюда следует, что пара $(\widehat{P}(K \cup N), \widehat{P}(K \cup N) \cap \widehat{P}(Y))$ $\left(\mathscr{M}_{1}, \mathscr{M}_{\alpha}\right)$-универсальна.

II) $\alpha$ - предельный ординал. Тогда возможны два варианта:

(1) существует такая точка $x \in X$, что для любой ее окрестности $U \subset X$ $U \cap Y \notin \cup_{\xi<\alpha} \mathscr{M}_{\xi}$

(2) такой точки не существует.

В первом случае, используя [21, 22.13 и 24.20$]$, можно построить такой компакт $B \subset X$, что $B \cap Y \notin \bigcup_{\xi<\alpha} \mathscr{M}_{\xi}$. Далее поступаем совершенно аналогично случаю $\alpha=\beta+1$.

Рассмотрим теперь вариант (2). Пусть $\left\{\alpha_{n}\right\}$ - возрастаюшая последовательность ординалов, сходящаяся к ординалу $\alpha$. Легко видеть, что тогда существует такое замкнутое в $X$ дискретное множество $\left\{x_{n}\right\}_{n=1}^{\infty} \subset X$, что для любого $n \in \mathbb{N}$ и любой окрестности $U$ точки $x_{n}$ имеем $U \cap Y \notin \mathscr{M}_{\alpha_{n}}$. Подходящим выбором метрики $d$ на $X$ можно добиться, чтобы $d\left(x_{n}, x_{m}\right) \geqslant 3$ для $n, m \in \mathbb{N}$. Используя [21, 22.13 и 24.20], для каждого $n \in 2 \mathbb{N}$ находим такой компакт $K_{n} \subset O\left(x_{n}, 1\right)$, что $K_{n} \cap Y \notin \mathscr{M}_{\alpha_{n}}$ и $K_{n} \cap Y$-плотно в $K_{n}$.

Легко видеть, что множества $N=\left\{x_{n} \mid n \in 2 \mathbb{N}+1\right\}$ и $B=N \cup \bigcup_{n \in 2 \mathbb{N}} K_{n}$ замкнуты в $X$, и дополнение $X \backslash B$ бесконечно. Более того, пара $(\widehat{P}(B), \widehat{P}(B \cap Y))$ $\left(\mathscr{M}_{1}, \mathscr{M}_{\alpha}\right)$-универсальна. Действительно, пространство $\widehat{P}(B)$ содержит множестBо

$$
M=\left\{\mu \in \widehat{P}(B) \mid \mu(N)=\frac{1}{2}, \mu\left(K_{2 n}\right)=2^{-n+1}, n \in \mathbb{N}\right\},
$$

которое гомеоморфно произведению $\widehat{P}(N) \times \prod_{n=1}^{\infty} \widehat{P}\left(K_{2 n}\right)$. При этом пара $(M, M \cap \widehat{P}(Y))$ гомеоморфна паре $\left(s \times \prod_{n=1}^{\infty} \widehat{P}\left(K_{2 n}\right), s \times \prod_{n=1}^{\infty} \widehat{P}\left(K_{2 n} \cap Z\right)\right)$, которая $\left(\mathscr{M}_{1}, \mathscr{M}_{\alpha}\right)$-предуниверсальна, поскольку для каждого $n \in \mathbb{N}$ пара $\left(\widehat{P}\left(K_{2 n}\right), \widehat{P}\left(K_{2 n} \cap Z\right)\right)$ сильно $\left(\mathscr{M}_{0}, \mathscr{M}_{\alpha_{2 n}}\right)$-универсальна (см. 2.5), и в силу 2.4 $\widehat{P}(N) \cong s$.

Из теоремы 2.4 следует, что для сепарабельного полного некомпактного метрического пространства $X$, пространство $\widehat{P}(X)$ гомеоморфно псевдовнутренности $s=(-1,1)^{\omega}$ гильбертова куба, которая, в свою очередь, гомеоморфна сепарабельному гильбертову пространству $\ell^{2}$. Ниже мы доказываем несепарабельный аналог этого утверждения. 
2.11. ТЕОРЕМА. Пусть $X$ - полное метрическое некомпактное пространство плотности А. Тогда пространство $\widehat{P}(X)$ гомеоморфно гильбертову пространству $\ell^{2}(A)=\left\{\left(x_{a}\right) \mid a \in A, x_{a} \in \mathbb{R}, \sum_{a \in A} x_{a}^{2}<\infty\right\}$ плотности $A$.

Для доказательства теоремы нам понадобятся два вспомогательных утверждения.

2.12. ЛЕмма. Если $X$ - выпуклое полнометризуемое AR-подмножество плотности $А$ в линейном топологическом пространстве, тогда произведение $X \times \ell^{2}$ гомеоморфно гильбертову пространству $\ell^{2}(A)$.

ДоказАтельство. Если кардинал $A$ счетен, то лемма следует из [23]. Так что далее мы предполагаем, что кардинал $A$ несчетен. Мы покажем, что любое замкнутое подмножество $K \subset X$ плотности $<A$, является $Z$-множеством в $X$. Пусть $\mathscr{U} \in \operatorname{cov}(X)$ - любое покрытие и $f: Q \rightarrow X$ отображение гильбертова кирпича. Очевидно, что множество $X \cap L$, где $L$ - линейная оболочка множества $K \cup f(Q)$ имеет плотность $<A$. Следовательно, существует точка $a \in X \backslash L$. Выберем $\varepsilon>0$ так, чтобы отображение $\bar{f}: Q \rightarrow X$, где $\bar{f}(q)=(1-\varepsilon) f(q)+\varepsilon a$ было $\mathscr{U}$-близким к $f$. Легко видеть, что $\bar{f}(Q) \cap K=\varnothing$. Таким образом $K-Z$-множество в $X$. Теперь лемма следует из предложения 6.2 [3].

2.13. ЛЕмма. Если $X$ - полное метрическое пространство плотности $A$, содержащее открыто-замкнутое счетное дискретное подмнохество, тогда пространство $\widehat{P}(X)$ гомеоморфно гильбертову пространству $\ell^{2}(A)$.

ДокаЗАТЕльство. Запишем $X=Y \cup N$, где $N$-счетное дискретное открыто-замкнутое подмножество $X$ и $Y=X \backslash N$. В пространстве $\widehat{P}(X)$ рассмотрим открытое вьпуклое подмножество

$$
\widehat{P}(X) \backslash(\widehat{P}(Y) \cup \widehat{P}(N))=\{\mu \in \widehat{P}(X) \mid 0<\mu(N)<1\},
$$

которое гомеоморфно произведению $(0,1) \times \widehat{P}(N) \times \widehat{P}(Y)$ посредством гомеоморфизма

$$
h:(0,1) \times \widehat{P}(N) \times \widehat{P}(Y) \rightarrow \widehat{P}(X) \backslash(\widehat{P}(Y) \cup \widehat{P}(N)),
$$

где $h(t, \mu, \eta)=t \mu+(1-t) \eta,(t, \mu, \eta) \in(0,1) \times \widehat{P}(N) \times \widehat{P}(Y)$. Поскольку пространство $\widehat{P}(N)$ гомеоморфно $\ell^{2}$ (см. теорему 2.4 ), то по лемме 2.12 произведение $(0,1) \times \widehat{P}(N) \times \widehat{P}(Y)$, а значит и пространство $\widehat{P}(X) \backslash(\widehat{P}(N) \cup \widehat{P}(Y))$ гомеоморфно $\ell^{2}(A)$.

Согласно [24, В1], если полное метрическое $A R$-пространство $E$ содержит такое сильное $Z$-множество $Z \subset E$, что дополнение $E \backslash Z$ гомеоморфно $\ell^{2}(A)$, тогда пространство $E$ также гомеоморфно $\ell^{2}(A)$. Напомним, что замкнутое множество $Z \subset E$ называется сильным $Z$-множеством, если для любого покрытия $\mathscr{U} \in \operatorname{cov}(E)$ существует такое $\mathscr{U}$-близкое к тождественному отображение $f: E \rightarrow E$, что $\operatorname{cl}_{E}(f(E)) \cap Z=\varnothing$. Итак, чтобы доказать, что пространство $\widehat{P}(X)$ гомеоморфно гильбертову пространству $\ell^{2}(A)$, нам достаточно доказать, что $\widehat{P}(N) \cup \widehat{P}(Y)$ является сильньм $Z$-множеством в $\widehat{P}(X)$. Для этого зафиксируем ограниченную единицей метрику $d$ на $X$ и функцию $\varepsilon: \widehat{P}(X) \rightarrow(0,1]$. Через $\widehat{d}$ обозначается метрика на $\widehat{P}(X)$, порожденная метрикой $d($ см. $[8, \S 4])$. 
Определим отображение $f: \widehat{P}(X) \rightarrow \widehat{P}(X)$ формулой $f(\mu)=(1-\varepsilon(\mu)) \mu+$ $\varepsilon(\mu) \eta_{0}$, где $\eta_{0}-$ любая мера вида $\eta_{0}=\frac{1}{2} \delta_{x}+\frac{1}{2} \delta_{y}, x \in N, y \in Y$. Легко видеть, что $\widehat{d}(f(\mu), \mu) \leqslant \varepsilon(\mu)$ и $\operatorname{cl}(f(\widehat{P}(X))) \cap(\widehat{P}(N) \cup \widehat{P}(Y))=\varnothing$, т.е. $\widehat{P}(N) \cup \widehat{P}(Y)-$ сильное $Z$-множество в $\widehat{P}(X)$.

ДоКАЗАТЕЛЬСТво ТЕОРЕмЫ 2.11. Итак, пусть $X$ - полное метрическое некомпактноепространство плотности $A$. Поскольку пространство $X$ - некомпактно, оно содержит замкнутое счетное дискретное подмножество $D \subset X$. Зафиксируем любой гомеоморфизм $h^{\prime}: \mathbb{N} \rightarrow D$ множества натуральных чисел $\mathbb{N}$ на $D$ и рассмотрим пространство $X \sqcup \mathbb{N}$ являюшееся дискретным объединением пространств $X$ и $\mathbb{N}$. Пусть $h: X \sqcup \mathbb{N} \rightarrow X$ - отображение, определенное формулой $h(x)=x$ для $x \in X$ и $h(n)=h^{\prime}(n)$ для $n \in \mathbb{N}$. Очевидно, что $h: X \sqcup \mathbb{N} \rightarrow X-$ совершенное (т.е. замкнутое с компактньми прообразами точек) сюръективное отображение. Согласно $[7,2.2]$, отображение $\widehat{P}(h): \widehat{P}(X \sqcup \mathbb{N}) \rightarrow \widehat{P}(X)$ также сюръективное и собственное. Кроме того, поскольку $\widehat{P}(h)$ - аффинное отображение между выпуклыми подмножествами локально выпуклых пространств, $\widehat{P}(h)$ является $C E$-отображением $[15, \S 7.1]$. Итак, мы установили, что пространство $\widehat{P}(X)$ является совершенным $C E$-образом пространства $\widehat{P}(X \sqcup \mathbb{N})$, гомеоморфного гильбертову пространству $\ell^{2}(A)$. Согласно $[3,4.1,4.2]$ пространство $\widehat{P}(X)$ также гомеоморфно $\ell^{2}(A)$.

\section{§3. Топология пространства $P_{d}(X)$ вероятностных дискретных мер}

Цель этого параграфа - получить топологическую классификацию пар вида $\left(P(X), P_{d}(Y)\right)$, где $X$ - компактное метрическое пространство, $Y$ - его всюду плотное $F_{\sigma \delta}$-подмножество, и $P_{d}(Y)$ - подпространство $\widehat{P}(Y)$, состоящее из вероятностных дискретных мер. Напомним, что мера $\mu \in \widehat{P}(X)$ назьвается дискретной, если $\mu(C)=1$ для некоторого счетного множества $C \subset X$. Если $f: X \rightarrow Y$ - отображение тихоновских пространств, тогда, очевидно, что $\widehat{P}(f)\left(P_{d}(X)\right) \subset P_{d}(Y)$. Таким образом, положив $P_{d}(f)=\widehat{P}(f) \mid P_{d}(X): P_{d}(X) \rightarrow P_{d}(Y)$ мы определяем подфунктор $P_{d}$ функтора $\widehat{P}$. Функтор $P_{d}$ обладает многими замечательными свойствами: например, он сохраняет сюръективность отображений, он монадичен на категории Тусһ и т. д. Мы не будем, однако, останавливаться на функториальных свойствах конструкции $P_{d}$, а будем основательно изучать лишь топологию пространств $P_{d}(X)$.

Начнем мы с борелевской классификации.

3.1. ПРЕДЛОЖЕНИЕ. Для любого тихоновского пространства $X$, пространство $P_{d}(X)$ является $F_{\sigma \delta}$-множеством в $\widehat{P}(X)$.

ДоКАЗАТЕЛЬСТво. Пусть $X$ - тихоновское пространство и $c X$ - любая его компактификация. Согласно $[7,2.4]$ мы можем рассматривать пространство $\widehat{P}(X)$ как подмножество компакта $P(c X)$, причем $P_{d}(X)=\widehat{P}(X) \cap P_{d}(c X)$. Предложение будет доказано, если мы покажем, что $P_{d}(c X)-F_{\sigma \delta}$-множество в $P(c X)$. Но, очевидно, что

$$
P_{d}(c X)=\bigcap_{n=1}^{\infty} \bigcup_{m=1}^{\infty} M_{n, m},
$$

где

$$
M_{n, m}=\left\{\mu \in P(c X)|\exists A \subset c X,| A \mid \leqslant m, \text { такое, что } \mu(A) \geqslant 1-\frac{1}{n}\right\} .
$$


Покажем, что для любых $n, m \in \mathbb{N}$ множество $M_{n, m}$ замкнуто в $P(c X)$. Пусть $\mu \in P(c X)$ - предельная точка множества $M_{n, m}$. Тогда существует направленность $\left\{\mu_{i}\right\}_{i \in \mathscr{I}} \subset M_{n, m}$, сходящаяся к мере $\mu$. Через $\exp _{m}(c X)$ мы обозначаем подпространство гиперпространства $\exp (c X)$, состояшее из множеств мошности $\leqslant m$. Для каждого $i \in \mathscr{I}$ зафиксируем такое $A_{i} \in \exp _{m}(c X)$, что $\mu_{i}\left(A_{i}\right) \geqslant$ $1-1 / n$. Поскольку пространство $\exp _{m}(c X)$ - компактно $[25, \mathrm{IV}, \S 4]$, то существует $A \in \exp _{m}(c X)$ - предельная точка направленности $\left\{A_{i}\right\}_{i \in \mathscr{I}}[26,3.1 .23]$. Покажем, что $\mu(A) \geqslant 1-1 / n$, т.е. $\mu \in M_{n, m}$. Поскольку мера $\mu$ регулярна, т.е. $\mu(A)=\inf \{\mu(U) \mid U \supset A$ - открытое подмножество $c X\}$, то нам достаточно доказать, что $\mu(U) \geqslant 1-1 / n-\varepsilon$ для любого $\varepsilon>0$ и любого открытого множества $U \subset c X$, содержашего множество $A$. Пусть $V \subset c X$ - такое открытое множество, что $A \subset V \subset \bar{V} \subset U$. Поскольку направленность $\left\{\mu_{i}\right\}_{i \in \mathscr{I}}$ сходится к мере $\mu$, то существует такое $i_{0} \in \mathscr{I}$, что $\mu_{i}(\bar{V})<\mu(\bar{V})+\varepsilon$ для любого $i \geqslant i_{0}$ [5, теорема II.2]. Поскольку $A$ - предельная точка направленности $\left\{A_{i}\right\}_{i \in \mathscr{I}}$, то сушествует такое $i \geqslant i_{0}$, что $A_{i} \subset V$. Тогда

$$
\mu(U) \geqslant \mu(\bar{V})>\mu_{i}(\bar{V})-\varepsilon \geqslant \mu_{i}\left(A_{i}\right)-\varepsilon \geqslant 1-\frac{1}{n}-\varepsilon .
$$

Если $Y \subset X$ - пара тихоновских пространств, тогда $P_{d}(Y)=P_{d}(X) \cap \widehat{P}(Y)$. Отсюда, из предложения 3.1 и [7, 2.30] следует

3.2. ПРЕДЛОЖЕНИЕ. Пусть $X$ - метрическое пространство, $Y \subset X-$ его подмноэсество и $\alpha \geqslant 2$ - счетный ординал. Если $Y \in \mathscr{M}_{\alpha}(X)$, тогда $P_{d}(Y) \in \mathscr{M}_{\alpha}(\widehat{P}(X))$.

Поскольку любая радоновская мера на метрическом пространстве имеет сепарабельный носитель, и любая радоновская мера на счетном пространстве является дискретной, то справедливо

3.3. ПРЕДЛОЖЕНИЕ. Если каждое сепарабельное подпространство метрического пространства $X$ счетно, тогда $P_{d}(X)=\widehat{P}(X)$. В частности, если борелевское подмножество $X$ полного метрического пространства не содержит канторова куба $2^{\omega}$, тогда $P_{d}(X)=\widehat{P}(X)$.

Для метрического пространства $X$ через $X^{(1)} \subset X$ мы обозначаем множество предельных точек $X$.

3.4. ПРЕДЛОЖЕНИЕ. Пусть $X$ - метрическое пространство и $Y \subset X-$ его всюду плотное подмножество. Если множество $Y^{(1)}$ предельных точек нигде не плотно в $Y$, тогда $\widehat{P}(X) \backslash P_{d}(Y)$ содержится в $\sigma Z$-множестве в $\widehat{P}(X)$. В противном случае, $P_{d}(Y)$ содержится в $\sigma Z$-множестве в $\widehat{P}(X)$.

ДокАЗАТЕЛЬСТво. Предположим, что множество $Y^{(1)}$ предельных точек нигде не плотно в $Y$. Тогда множество $Y \backslash Y^{(1)}=X \backslash Y^{(1)}$ изолированных точек открыто и всюду плотно в $X$. Из доказательства предложения 2.3 следует, что множество $\widehat{P}(X) \backslash \widehat{P}\left(Y \backslash Y^{(1)}\right)$ содержится в $\sigma Z$-множестве в $\widehat{P}(X)$. Но согласно предложению $3.3 P_{d}\left(Y \backslash Y^{(1)}\right)=\widehat{P}\left(Y \backslash Y^{(1)}\right)$. Следовательно, множество

$$
\widehat{P}(X) \backslash P_{d}(Y) \subset \widehat{P}(X) \backslash P_{d}\left(Y \backslash Y^{(1)}\right)=\widehat{P}(X) \backslash \widehat{P}\left(Y \backslash Y^{(1)}\right)
$$


содержится в $\sigma Z$-множестве в $\widehat{P}(X)$.

Теперь предположим, что множество $Y^{(1)}$ где-то плотно и покажем, что тогда $P_{d}(Y)$ содержится в $\sigma Z$-множестве в $\widehat{P}(X)$. Положим $F=\mathrm{cl}_{X}\left(Y \backslash Y^{(1)}\right)$. Согласно предположению, $F \neq X$. Пусть $G=\operatorname{cl}_{X}(X \backslash F)$. Тогда

$$
P_{d}(Y) \subset \widehat{P}(F) \cup\left(\bigcup_{n=1}^{\infty} A_{n}\right)
$$

где

$$
A_{n}=\left\{\mu \in \widehat{P}(X) \mid \exists x \in G \text { такое, что } \mu(\{x\}) \geqslant \frac{1}{n}\right\} .
$$

Из доказательства предложения 2.2 следует, что $\widehat{P}(F)-Z$-множество в $\widehat{P}(X)$, а из доказательства предложения 3.1 , что $A_{n}$ - замкнутые подмножества в $\widehat{P}(X)$. Кроме того, нетрудно убедиться, что для любого $n \in \mathbb{N}$ множество

$$
\widehat{P}(X) \backslash A_{n}=\left\{\mu \in \widehat{P}(X) \mid \forall x \in G \mu(x)<\frac{1}{n}\right\}
$$

вьпукло и всюду плотно в $\widehat{P}(X)$. Следовательно, $A_{n}$ - замкнутое гомотопически пренебрежимое множество в $\widehat{P}(X)$, откуда следует, что $A_{n}-Z$-множество в $\widehat{P}(X)$. Таким образом, множество $P_{d}(Y)$ содержится в $\sigma Z$-множестве

$$
\widehat{P}(F) \cup\left(\bigcup_{n=1}^{\infty} A_{n}\right) \subset \widehat{P}(X) .
$$

Основным результатом этого параграфа является следуюшая

3.5. Теорема. Пусть $X$ - бесконечный метрический компакт и $Y \subset X$ - всюду плотное собственное $F_{\sigma \delta}$-подмножество $X$. Пара $\left(P(X), P_{d}(Y)\right)$ гомеоморфна

(1) паре $(Q, s)$, если $Y$ - счетное $G_{\delta}$-множсество в $X$;

(2) паре $\left(Q, \Omega_{2}(Q)\right)$, если $Y$ не является счетным $G_{\delta}$-множеством в $X$ и множество $Y^{(1)}$ предельных точек дде-то плотно в $X$;

(3) паре $\left(Q, Q \backslash \Lambda_{2}(Q)\right)$, если $Y$ не является счетным $G_{\delta}$-мнохсеством в $X$ и множсество $Y^{(1)}$ предельных точек нигде не плотно в $X$.

Для доказательства нам понадобится

3.6. Лемма. Пусть $D$ - счетное всюду плотное множество в канторовом кубе $C=\{0,1\}^{\omega}$. Пара $\left(P(C), P_{d}(D)\right)\left(\mathscr{M}_{0}, \mathscr{M}_{2}\right)$-предуниверсальна.

Доказательство. Рассмотрим отображение $h:[0,1] \rightarrow P(\{0,1\})$, где $h(t)=$ $\frac{t}{2} \delta_{0}+\left(1-\frac{t}{2}\right) \delta_{1}, t \in[0,1]$, а также отображение $H:[0,1]^{\omega} \rightarrow P\left(\{0,1\}^{\omega}\right)$, где $H\left(\left(t_{i}\right)_{i=1}^{\infty}\right)=\bigotimes_{i=1}^{\infty} h\left(t_{i}\right)$ - тензорное произведение мер $h\left(t_{i}\right) \in P(\{0,1\})$. Легко видеть, что

$$
\begin{aligned}
H^{-1}\left(P_{d}\left(\{0,1\}^{\omega}\right)\right) & =W([0,1], 0) \\
& =\left\{\left(t_{i}\right)_{i=1}^{\infty} \mid t_{i} \neq 0 \text { для конечного числа индексов } i\right\} .
\end{aligned}
$$


Обозначим пару $\left([0,1]^{\omega}, W([0,1], 0)\right)$ через $(Q, \sigma)$ и рассмотрим отображение $f: Q^{\omega} \rightarrow P(C)$, где

$$
f\left(\left(q_{i}\right)_{i=1}^{\infty}\right)=\sum_{i=1}^{\infty} 2^{-i} H\left(q_{i}\right), \quad\left(q_{i}\right)_{i=1}^{\infty} Q^{\omega}
$$

Легко видеть, что $f^{-1}\left(P_{d}(C)\right)=\sigma^{\omega}$. Поскольку пара $\left(Q^{\omega}, \sigma^{\omega}\right)\left(\mathscr{M}_{0}, \mathscr{M}_{2}\right)$-предуниверсальна, то пара $\left(P(C), P_{d}(C)\right)$ также $\left(\mathscr{M}_{0}, \mathscr{M}_{2}\right)$-предуниверсальна.

ДОКАЗАТЕЛЬСТВо ТЕОРЕМЫ 3.5. Если множество $Y$ счетно, тогда $P_{d}(Y)=$ $\widehat{P}(Y)$ и утверждение теоремы следует из 2.7. Поэтому далее мы предполагаем, что $Y$ несчетно. Тогда по $[18, \S 37] Y$ содержит топологическую копию $C \subset Y$ канторова куба. В этом случае лемма 3.7 позволяет утверждать, что пара $\left(\widehat{P}(X), P_{d}(Y)\right)$ $\left(\mathscr{M}_{0}, \mathscr{M}_{2}\right)$-предуниверсальна.

Теперь наша теорема немедленно следует из 1.1, 1.2, 3.2 и 3.4 .

В связи с результатами этого параграфа интересньм представляется

3.7. Вопрос. Пусть $X \subset C$-подмножество канторова куба. Может ли пространство $P_{d}(X)$ быть $\mathscr{A}_{2}$-универсальным?

\section{§4. Топология пространства $P_{c}(X)$ непрерывных вероятностных мер}

В этом параграфе мы изучаем топологию подпространства $P_{c}(X) \subset \widehat{P}(X)$, состоящего из непрерывных мер. Напомним, что мера $\mu \in \widehat{P}(X)$ называется непрерывной, если $\mu(\{x\})=0$ для каждой точки $x \in X$. Легко видеть, что множество $P_{c}(X)$ выпукло в $\widehat{P}(X)$.

Отметим, что непрерывные меры существуют не на всех пространствах. Напомним, что точка $x$ пространства $X$ называется точкой конденсачии, если каждая ее окрестность несчетна, иначе говоря, если пространство $X$ не является локально счетным в точке $x[18, \S 23 . \mathrm{III}]$. Множество точек конденсации пространства $X$ обозначается через $X \odot$. Хорошо известно, что для метрического сепарабельного пространства $X$ множество $X \odot$ замкнуто в $X$, а дополнение $X \backslash X \odot$ счетно. Следовательно, множество $X{ }^{\odot}$ непусто тогда и только тогда, когда пространство $X$ несчетно.

Непосредственно из определения носителя меры следует, что $\operatorname{supp}(\mu) \subset X{ }^{\odot}$ для любой непрерывной меры $\mu \in P_{c}(X)$. Таким образом, справедливо

4.1. ПРЕДЛОЖЕНИЕ. Для любого метрического пространства $Х$ имеем $P_{c}(X)=P_{c}\left(X^{\odot}\right)$.

Отметим, что подпространство $X^{\odot} \subset X$ всюду несчетно (т.е. любое непустое открытое подмножество $X^{\odot}$ несчетно). Более того, $X=X^{\odot}$ тогда и только тогда, когда $X$ - всюду несчетное пространство. Поэтому, ввиду предложения 4.1 , при рассмотрении пространств $P_{c}(X)$, достаточно ограничиться всюду несчетными пространствами $X$.

Напомним, что аналитическим пространством назьвается любой непрерывный метризуемый образ пространства $\mathbb{N}^{\omega}$ иррациональных чисел. Хорошо известно, что борелевское подмножества метрических компактов являются аналитическими пространствами [21]. 
4.2. ПРЕДЛОЖЕНИЕ. Если $X$ - аналитическое всюду несчетное пространство, тогда $P_{c}(X)$ всюду плотное выпуклое подмножсество в $\widehat{P}(X)$.

ДокАЗАтЕльство. Выпуклость множества $P_{c}(X)$ очевидна. Покажем его всюду плотность в $\widehat{P}(X)$. Для этого зафиксируем меру $\mu_{0} \in \widehat{P}(X)$ и ее окрестность $U \subset \widehat{P}(X)$. Поскольку множество $P_{\omega}(X)$ мер с конечными носителями всюду плотно в $\widehat{P}(X)$, то сушествует мера

$$
\eta=\sum_{i=1}^{n} t_{i} \delta_{x_{i}} \in U \cap P_{\omega}(X),
$$

где $t_{i}>0, x_{i} \in X$ и $\sum_{i=1}^{n} t_{i}=1$. Несложно построить такие окрестности $U_{i} \subset X$ точек $x_{i}$, что для любых мер $\mu_{i} \in \widehat{P}\left(U_{i}\right) \subset \widehat{P}(X)$ мера $\sum_{i=1}^{n} t_{i} \mu_{i}$ близка к $\eta$ и принадлежит окрестности $U$. Поскольку пространство $X$ аналитично и всюду несчетно, то же верно для множеств $U_{i}$. Тогда, согласно теореме 29.1 [21], каждое множество $U_{i}$ содержит топологическую копию $K_{i}$ канторова куба $2^{\omega}$. Очевидно, что на канторовом кубе сушествует непрерывная мера (например инвариантная мера Хаара, ведь $2^{\omega}$ - компактная топологическая группа). Следовательно, на каждом $K_{i}$ сушествует непрерывная мера $\mu_{i} \in P_{c}\left(K_{i}\right) \subset \widehat{P}(X)$. Тогда мера $\mu=\sum_{i=1}^{n} t_{i} \mu_{i}$ тоже непрерывна и принадлежит окрестности $U$ меры $\mu_{0}$. То есть множество $P_{c}(X)$ всюду плотно в $\widehat{P}(X)$.

4.3. ПРЕДЛОЖЕНИЕ. Для любого тихоновского пространства $X$ пространство $P_{c}(X)$ является $G_{\delta}$-мнохеством в $\widehat{P}(X)$.

ДокАЗАТЕльство. Рассуждая аналогично как при доказательстве предложения 3.1 , можно ограничится случаем, когда $X$ - компакт. Очевидно, что

$$
P(X) \backslash P_{c}(X)=\bigcup_{n=1}^{\infty} M_{n}
$$

где

$$
M_{n}=\left\{\mu \in P(X) \mid \exists x \in X \operatorname{c} \mu(\{x\}) \geqslant \frac{1}{n}\right\} .
$$

Повторяя аргументы предложения 3.1, приходим к выводу, что каждое множество $M_{n}$ замкнуто в $P(X)$. Следовательно, $P_{c}(X)-G_{\delta}$-множество в $P(X)$.

Если $Y \subset X$ - пара тихоновских пространств, тогда $P_{c}(Y)=P_{c}(X) \cap \widehat{P}(Y)$. Отсюда, из предложения 4.3 и $[7,2.30]$ следует

4.4. ПРЕДЛОЖЕНИЕ. Пусть $X$ - метрическое пространство, $Y \subset X$ его подмножсество и $\alpha \geqslant 1$ - счетный ординал. Если $Y \in \mathscr{M}_{\alpha}(X)$, тогда $P_{c}(Y) \in \mathscr{M}_{\alpha}(\widehat{P}(X))$.

Повторяя аргументацию теоремы 2.4, можно доказать следующий факт.

4.5. Теорема. Пусть $X$ - метрический компакт и $Y \subset X-$ его всюду плотное всюду несчетное $G_{\delta}$-подмнохсество. Тогда пара $\left(P(X), P_{c}(Y)\right)$ гомеоморфна паре $(Q, s)$.

Теперь рассмотрим вопрос, когда пространство $P_{c}(X)$ является $\sigma Z$-множеством. Поскольку всегда $P_{c}(Y) \subset \widehat{P}(Y)$, то из предложения 2.2 следует 
4.6. ПРЕДЛОЖЕНИЕ. Пусть $Y \subset X-$ пара метрических пространств. Если пространство $Y$ не является бэровским, то $P_{c}(Y)$ содержится в $\sigma Z$-множсестве пространства $\widehat{P}(X)$.

Для пространств непрерывных вероятностных мер сушествует также аналог предложения 2.3 .

4.7. ПРЕДЛОЖЕНИЕ. Пусть $X$-метрическое сепарабельное пространство и $Y$ - всюду плотное аналитическое всюду несчетное подпространство в X. Если пространство $Y$ - бәровское, тогда $\widehat{P}(X) \backslash P_{c}(Y)$ содержится в бZ-подмножестве пространства $\widehat{P}(X)$.

ДокАЗАТЕЛЬСтво. Поскольку пространство $Y$ аналитично, то по теореме 29.5 [21] $Y=G \cup F$, где $G$ - абсолютное $G_{\delta}$-множество и $F$ - множество первой категории в $Y$. Поскольку $Y$ - бэровское пространство, множество $G$ - всюду плотно в $Y$ (и, следовательно, в $X$ ). Отметим, что пространство $G$ всюду несчетно, иначе, по теореме Бэра, $G$ содержало бы изолированную точку, что исключено ввиду всюду плотности $G$ во всюду несчетном пространстве $Y$. По предложениям 4.2 и $4.4, P_{c}(G)$ - вьпуклое всюду плотное $G_{\delta}$-подмножество в $\widehat{P}(X)$. Тогда дополнение $\widehat{P}(X) \backslash P_{c}(G)$ является искомьм $\sigma Z$-множеством, содержащим $\widehat{P}(X) \backslash P_{c}(Y)$.

4.8. ТЕОРемА. Пусть $X$-метрический компакт и $Y \subset X-$ всюду плотное всюду несчетное подмножество. Предположим, что $Y \notin \mathscr{M}_{2} \cap \mathscr{A}_{2}$ и $Y \in$ $\mathscr{M}_{\alpha} \backslash \bigcup_{\xi<\alpha} \mathscr{M}_{\alpha}$ для некоторого $\alpha \geqslant 2$. Тогда пара $\left(P(X), P_{c}(Y)\right)$ гомеоморфна

(1) паре $\left(Q, Q \backslash \Lambda_{\alpha}(Q)\right)$, если $Y$ - бэровское пространство и

(2) паре $\left(Q, \Omega_{\alpha}(Q)\right)$ в противном случае.

ДоказАТЕльство. Аналогично, как и при доказательстве теоремы 2.7, достаточно доказать $\left(\mathscr{M}_{0}, \mathscr{M}_{\alpha}\right)$-предуниверсальность пары $\left(P(X), P_{c}(Y)\right)$ и затем использовать теоремы 1.1, 1.2 и предложения $4.4,4.6$ и 4.7.

Рассмотрим три случая.

I) $\alpha=2$. Тогда $Y \in \mathscr{M}_{2} \backslash \mathscr{A}_{2}$. Зафиксируем любое подмножество $A \subset 2^{\omega}$ класса $\mathscr{M}_{2} \backslash \mathscr{M}_{1}$. Поскольку $Y \notin \mathscr{A}_{2}$, мы можем использовать теорему Луво и Сан-Ремо $[21,28.19]$, согласно которой, сушествует такое вложение $e: 2^{\omega} \times 2^{\omega} \rightarrow X$, что $e^{-1}(Y)=A \times 2^{\omega}$. Применяя к этому вложению функтор $P$, получим вложение $P(e): P\left(2^{\omega} \times 2^{\omega}\right) \rightarrow P(X)$, причем $P(e)^{-1}\left(P_{c}(Y)\right)=P_{c}\left(A \times 2^{\omega}\right)$. Зафиксируем любую непрерывную вероятностную меру $\mu_{0}$ на канторовом кубе $2^{\omega}$ и рассмотрим отображение $f: P\left(2^{\omega}\right) \rightarrow P\left(2^{\omega} \times 2^{\omega}\right)$, ставящее в соответствие мере $\mu \in P\left(2^{\omega}\right)$ меру $f(\mu)=\mu \otimes \mu_{0}$ (тензорное произведение мер $\mu$ и $\left.\mu_{0}\right)$. Очевидно, что $f\left(P\left(2^{\omega}\right)\right) \subset$ $P_{c}\left(2^{\omega} \times 2^{\omega}\right)$ и $f^{-1}\left(P_{c}\left(A \times 2^{\omega}\right)\right)=f^{-1}\left(\widehat{P}\left(A \times 2^{\omega}\right)\right)=\widehat{P}(A)$. Следовательно, для отображения $P(e) \circ f: P\left(2^{\omega}\right) \rightarrow P(X)$ имеем: $(P(e) \circ f)^{-1}\left(P_{c}(Y)\right)=\widehat{P}(A)$. Поскольку $A \in \mathscr{M}_{2} \backslash \mathscr{M}_{1}$, то пара $\left(P\left(2^{\omega}\right), \widehat{P}(A)\right)\left(\mathscr{M}_{0}, \mathscr{M}_{2}\right)$-универсальна, согласно предложению 2.5. Тогда пара $\left(P(X), P_{c}(Y)\right)\left(\mathscr{M}_{0}, \mathscr{M}_{2}\right)$-предуниверсальна.

II) $\alpha=\beta+1>2$ - непредельный ординал. В этом случае, $Y \notin \mathscr{M}_{\beta}$. Зафиксируем любое подмножество $A \subset 2^{\omega}$ класса $\mathscr{A}_{\beta} \backslash \mathscr{M}_{\beta}$ и, используя теорему Луво и Сан-Ремо [21, 28.19], найдем такое вложение $e: 2^{\omega} \times 2^{\omega} \rightarrow X$, что $e^{-1}(Y)=A \times 2^{\omega}$. Повторяя далее рассуждения случая $\alpha=2$, показываем, что пара $\left(P(X), P_{c}(Y)\right.$ $\left(\mathscr{M}_{0}, \mathscr{M}_{\alpha}\right)$-предуниверсальна. 
III) $\alpha$ - предельный ординал. Зафиксируем любую последовательность ординалов $\left\{\alpha_{n}\right\}$ с $2<\alpha_{n}<\alpha$ и $\sup _{n \in \mathbb{N}} \alpha_{n}=\alpha$. Для каждого $n \in \mathbb{N}$ выберем подмножество $A_{n} \subset 2^{\omega}$ класса $\mathscr{A}_{\alpha_{n}} \backslash \mathscr{M}_{\alpha_{n}}$. Повторяя аргументы предыдушего случая, для каждого $n \in \mathbb{N}$ построим такое отображение $f_{n}: P\left(2^{\omega}\right) \rightarrow P(X)$, что $f_{n}^{-1}\left(P_{c}(Y)\right)=\widehat{P}\left(A_{n}\right)$. Тогда отображение $f: P\left(2^{\omega}\right)^{\mathbb{N}} \rightarrow P(X)$, определенное формулой $f\left(\left(\mu_{n}\right)_{n \in \mathbb{N}}\right)=\sum_{n=1}^{\infty} 2^{-n} f_{n}\left(\mu_{n}\right)$, имеет следуюшее свойство:

$$
f^{-1}\left(P_{c}(Y)\right)=\prod_{n \in \mathbb{N}} \widehat{P}\left(A_{n}\right)
$$

Поскольку для каждого $n$ пара $\left(P\left(2^{\omega}\right), \widehat{P}\left(A_{n}\right)\right)\left(\mathscr{M}_{0}, \mathscr{M}_{\alpha_{n}}\right)$-универсальна, то произведение

$$
\left(\prod_{n \in \mathbb{N}} P\left(2^{\omega}\right), \prod_{n \in \mathbb{N}} \widehat{P}\left(A_{n}\right)\right)
$$

является $\left(\mathscr{M}_{0}, \mathscr{M}_{\alpha}\right)$-универсальной парой. Следовательно, пара $\left(P(X), P_{c}(Y)\right.$ $\left(\mathscr{M}_{0}, \mathscr{M}_{\alpha}\right)$-предуниверсальна.

Отметим, что теоремы 4.5 и 4.8 дают исчерпьваюшее описание топологии пространства $P_{c}(X)$ для всех сепарабельных абсолютных борелевских пространств, не принадлежащих классу $\left(\mathscr{M}_{2} \cap \mathscr{A}_{2}\right) \backslash \mathscr{M}_{1}$.

4.9. ПроБлема. Описать топологию пространства $P_{c}(X)$ для несчетных пространств $X \in\left(\mathscr{M}_{2} \cap \mathscr{A}_{2}\right) \backslash \mathscr{M}_{1}$.

\section{§5. Топология пространства вероятностных мер с конечными носителями}

Напомним, что для пространства $X$ пространство всех вероятностных мер с конечными носителями обозначается через $P_{\omega}(X) \subset \widehat{P}(X)$. Цель этого параграфа - найти необходимые и достаточные условия, при которых пара $\left(\widehat{P}(X), P_{\omega}(X)\right)$ гомеоморфнапаре $\left(\ell^{2}(A), \ell_{f}^{2}(A)\right)$, где $\ell^{2}(A)$ - стандартное гильбертово пространство плотности $A$, и

$$
\ell_{f}^{2}(A)=\left\{\left(x_{a}\right)_{a \in A} \in \ell^{2}(A) \mid x_{a} \neq 0 \text { для конечного числа индексов } a\right\} \subset \ell^{2}(A)
$$

- подпространство, состоящее из финитных “последовательностей".

Согласно [27] пара $\left(\ell^{2}(A), X\right)$ гомеоморфна паре $\left(\ell^{2}(A), \ell_{f}^{2}(A)\right)$ тогда и только тогда, когда выполнены следуюшие условия:

(1) множество $X$ представляется в виде счетного объединения конечномерных локально-компактных замкнутых подмножеств $\ell^{2}(A)$;

(2) для любого покрытия $\mathscr{U} \in \operatorname{cov}\left(\ell^{2}(A)\right)$, любого конечномерного локально компактного замкнутого множества $Z \subset \ell^{2}(A)$ и его замкнутого подмножества $F \subset Z \cap X$ сушествует такое замкнутое вложение $e: Z \rightarrow \ell^{2}(A)$, что $e(Z) \subset X, e \mid F=$ id $\mid F$ и $(e$, id $\mid Z) \prec \mathscr{U}$. 
Известно [12], [6], что для метрического компакта $X$ и его подмножества $Y$, пара $\left(P(X), P_{\omega}(Y)\right)$ гомеоморфна паре $(Q, \sigma)$ тогда и только тогда, когда множество $Y$ представляется в виде счетного объединения конечномерных компактов. Напомним, что

$$
\sigma=\left\{\left(q_{i}\right)_{i=1}^{\infty} \in(-1,1)^{\omega} \mid q_{i} \neq 0 \text { для конечного числа индексов } i\right\} \subset Q .
$$

5.1. ТЕОРемА. Пусть $X$ - полное метрическое некомпактное пространство плотности $А$ и $Y \subset X-$ его всюду плотное подмножество. Пара $\left(\widehat{P}(X), P_{\omega}(Y)\right)$ гомеоморфна паре $\left(\ell^{2}(A), \ell_{f}^{2}(A)\right)$ тогда и только тогда, когда мнохество $Y$ представляется в виде счетного объединения конечномерных локально компактных замкнутых подмножеств $X$.

ДоКАЗАТЕЛЬСтво. По теореме 2.11 , пространство $\widehat{P}(X)$ гомеоморфно гильбертову пространству $\ell^{2}(A)$. Если пара $\left(\widehat{P}(X), P_{\omega}(Y)\right)$ гомеоморфна паре $\left(\ell^{2}(A), \ell_{f}^{2}(A)\right)$, то, поскольку множество $Y$ - замкнуто в $P_{\omega}(X), Y$ представляется в виде счетного объединения конечномерных локально компактных замкнутых подмножеств $X$. Далее теорема следует из лемм 5.2 и 5.3 .

5.2. ЛЕмма. Пусть $X$ - полное метрическое пространство и $Y \subset X-$ подмножество, представляемое в виде счетного обвединения конечномерных локально компактных замкнутых подмножеств $X$. Тогда множество $P_{\omega}(Y)$ представляется в виде счетного обвединения конечномерных локально компактных замкнутых подмножеств $\widehat{P}(X)$.

ДокаЗАтельство. Пусть $Y=\bigcup_{n=1}^{\infty} Y_{n}$, где $Y_{n}, n \in \mathbb{N},-$ конечномерные локально компактные замкнутые подмножества $X$. Очевидно, что

$$
P_{\omega}(Y)=\bigcup_{n, m=1}^{\infty} P_{n}\left(Y_{m}\right) \backslash P_{n-1}\left(Y_{m}\right)
$$

(мы полагаем $P_{0}\left(Y_{m}\right)=\varnothing$ ), и для любых $n, m \in \mathbb{N}$

$$
P_{n}\left(Y_{m}\right) \backslash P_{n-1}\left(Y_{m}\right)=\bigcup_{k=1}^{\infty} A_{k},
$$

где

$$
\begin{aligned}
A_{k}=\left\{\mu \in P_{n}\left(Y_{m}\right) \backslash P_{n-1}\left(Y_{m}\right) \mid \mu\right. & =\sum_{i=1}^{n} t_{i} \delta\left(y_{i}\right), \\
\text { где } t_{i} & \left.\geqslant \frac{1}{k} \text { и } d\left(y_{i}, y_{j}\right) \geqslant \frac{1}{k} \text { для } 1 \leqslant i \neq j \leqslant n\right\}
\end{aligned}
$$

(здесь $d$ - метрика на $X$ ). Легко видеть, что каждое множество $A_{k}$ является конечномерным локально компактным замкнутым подмножеством в $\widehat{P}(X)$. 
5.3. Лемма. Пусть $X$ - полное метрическое некомпактное пространство и $Y \subset X-$-гго всюду плотное подмнохсество. Тогда для любого покрытия $\mathscr{U} \in \operatorname{cov}(\widehat{P}(X))$, любого конечномерного локально компактного замкнутого множества $Z \subset \widehat{P}(X)$ и его замкнутого подмножества $F \subset Z \cap P_{\omega}(Y)$ существует такое замкнутое вложение $е: Z \rightarrow \widehat{P}(X)$, ито $е(Z) \subset P_{\omega}(Y)$, $e|F=\operatorname{id}| F u(e, \operatorname{id} \mid Z) \prec \mathscr{U}$.

ДокАЗАТЕЛЬСТво. Зафиксируем полную ограниченную метрику $d$ на $X$, покрытие $\mathscr{U} \in \operatorname{cov}(\widehat{P}(X))$, конечномерное локально компактное замкнутое множество $Z \subset \widehat{P}(X)$ и его замкнутое подмножество $F \subset Z \cap P_{\omega}(Y)$. Через $\widehat{d}$ обозначаем метрику на $\widehat{P}(X)$, порожденную метрикой $d$.

Поскольку тождественное вложение $Z \backslash F \hookrightarrow \widehat{P}(X) \backslash F$ совершенно, то согласно $[28,4.1]$ сушествует такое покрытие $\mathscr{U}^{\prime} \in \operatorname{cov}(\widehat{P}(X) \backslash F)$, что любое $\mathscr{U}^{\prime}$-близкое к тождественному вложению отображение $g: Z \backslash F \rightarrow \widehat{P}(X) \backslash F$ является совершенным. Пусть $\mathscr{V} \in \operatorname{cov}(\widehat{P}(X) \backslash F)$ - такое покрытие, состоящее из выпуклых открытых множеств, что $\operatorname{St}(\mathscr{V}) \prec \mathscr{U}, \operatorname{St}(\mathscr{V}) \prec \mathscr{U}^{\prime}$ и $\operatorname{St}(\mathscr{V}) \prec\{O(\mu, \widehat{d}(\mu, F) / 2) \mid$ $\mu \in \widehat{P}(X) \backslash F\}$.

Мы построим сперва $\mathscr{V}$-близкое к тождественному вложению отображение $g: Z \backslash F \rightarrow P_{\omega}(Y) \backslash F . \quad$ Пусть $\mathscr{V}^{\prime} \in \operatorname{cov}(\widehat{P}(X) \backslash F)$ - такое локально конечное покрытие, что $\operatorname{St}\left(\mathscr{V}^{\prime}\right) \prec \mathscr{V}$, и $\left\{\lambda_{V}: \widehat{P}(X) \backslash F \rightarrow[0,1]\right\}_{V \in \mathcal{V}^{\prime}}-$ разбиение единицы, подчиненное покрытию $\mathscr{V}^{\prime}$. Для каждого элемента $V \in \mathscr{V}^{\prime}$ зафиксируем меру $\mu_{V} \in V \cap P_{\omega}(Y)$ (здесь мы используем плотность множества $P_{\omega}(Y)$ в $\widehat{P}(X))$. Наконец, определим отображение $g: Z \backslash F \rightarrow P_{\omega}(Y)$ формулой $g(z)=\sum_{V \in \mathcal{V}^{\prime}} \lambda_{V}(z) \cdot \mu_{V}$.

Несложно убедиться, что отображение $g \mathscr{V}$-близко к тождественному вложению $Z \backslash F \hookrightarrow \widehat{P}(X)$. Поскольку $\operatorname{St}(\mathscr{V}) \prec\{O(\mu, \widehat{d}(\mu, F) / 2) \mid \mu \in \widehat{P}(X) \backslash F\}$ и $\operatorname{St}(\mathscr{V}) \prec \mathscr{U}^{\prime}$, то $g(Z \backslash F) \subset P_{\omega}(Y) \backslash F$ и отображение $g: Z \backslash F \rightarrow P_{\omega}(Y) \backslash F$ совершенно. Более того, каждая точка $\mu \in g(Z \backslash F)$ обладает такой окрестностью $U \subset \widehat{P}(X)$, что $U \cap g(Z \backslash F) \subset P_{n}(Y)$ для некоторого $n \in \mathbb{N}$. Действительно, из конструкции отображения $g$, для каждой точки $y \in g(Z \backslash F) g^{-1}(y)$ - компакт, обладаюший окрестностью $V$ с $g(V) \subset P_{n}(Y)$ для некоторого $n \in \mathbb{N}$. Поскольку отображение $g$ - замкнуто, сушествует такая окрестность $U \subset \widehat{P}(X)$ точки $y$, что $g^{-1}(U) \subset V$ (см. [15, 1.1.10]). Тогда $U \subset g(V) \subset P_{n}(Y)$.

Пусть $\mathscr{W} \in \operatorname{cov}(g(Z \backslash F))$ - такое локально конечное покрытие, что $\mathscr{W} \prec \mathscr{V}$ и для каждого элемента $W \in \mathscr{W}$, его замькание $\bar{W}$ компактно и содержится в $P_{n(W)}(Y)$ для некоторого $n(W) \in \mathbb{N}$. Пусть $\left\{\lambda_{W}: g(Z \backslash F) \rightarrow[0,1]\right\}_{W \in \mathscr{W}}$ - разбиение единицы, подчиненное покрытию $\mathscr{W}$. Поскольку отображение $g: Z \backslash F \rightarrow P_{\omega}(Y) \backslash F$ собственно, а пространство $Z$ конечномерно, то для каждого $W \in \mathscr{W}$ прообраз $g^{-1}(\bar{W})$ является конечномерньм компактом. Следовательно, существует конечное множество $m(W)$ и вложение $e_{W}: g^{-1}(\bar{W}) \rightarrow P(m(W))$ такое, что для любых различных точек $z_{1}, z_{2} \in g^{-1}(\bar{W})$ их образы $e\left(z_{1}\right), e\left(z_{2}\right)$ различаются более чем по $2 n(W)+1$ координатах. Положим $D=\bigsqcup_{W \in \mathscr{W}} m(W)$ и определим отображение $e^{\prime}: Z \backslash F \rightarrow P_{\omega}(D)$ формулой

$$
e^{\prime}(z)=\sum_{W \in \mathscr{W}} \lambda_{W}(g(z)) e_{W}(z), \quad z \in Z \backslash F
$$


Несложно убедиться, что существует инъекция $i: D \rightarrow Y$, которая порождает инъекцию $P_{\omega}(i): P_{\omega}(D) \rightarrow P_{\omega}(Y)$.

Пусть $\mathscr{W}^{\prime} \in \operatorname{cov}(g(Z \backslash F))$ - такое покрытие, что $\operatorname{St}\left(\mathscr{W}^{\prime}\right) \prec \mathscr{W}$ и пусть $\varepsilon: g(Z \backslash F) \rightarrow(0,1]$ - такая функция, что для любого $\mu \in g(Z \backslash F) O_{\widehat{d}}(\mu, \varepsilon(\mu)) \subset W$ для некоторого $W \in \mathscr{W}^{\prime}$. Искомое вложение $e: Z \rightarrow \widehat{P}(X)$ определим следуюшим образом:

$$
e(z)= \begin{cases}z, & \text { если } z \in F \\ (1-\varepsilon(g(z))) \cdot g(z)+\varepsilon(g(z)) \cdot P_{\omega}(i) \circ e^{\prime}, & \text { если } z \in Z \backslash F .\end{cases}
$$

Легко видеть, что $(e \mid Z \backslash F, g) \prec \mathscr{W}^{\prime} \prec \mathscr{W} \prec \mathscr{V}$. Следовательно, отображение $e: Z \backslash F \rightarrow \widehat{P}(X) \backslash F$ - собственное. Нетрудно убедиться также, что отображение $e$ - инъективно. Поскольку собственные отображения замкнуты, то $e$ - вложение. Кроме того, из построения отображения $е$ ясно, что $e \mid F=\mathrm{id}, e(Z) \subset P_{\omega}(Y)$ и $(e, \mathrm{id}) \prec \mathscr{U}$.

\section{Список литературы}

1. Банах Т. О., Радул Т. М. Про функтор ймовірносних радонівських мір // Доповіді АН України. 1994. № 8. С. 16-20.

2. Toruńczyk $H$. On $C E$-images of the Hilbert cube and characterization of $Q$-manifilds // Fund. Math. 1980. V. 106. P. 31-40.

3. Toruńczyk H. Characterizing Hilbert space topology // Fund. Math. 1981. V. 111. P. 247-262.

4. Bestvina M., Mogilski J. Characterizing certain incomplete infinite-dimensional absolute retracts // Michigan Math. J. 1986. V. 33. № 3. P. 291-313.

5. Варадарайн В. С. Меры на топологических пространствах // Матем. сб. 1961. Т. 55. № 1. C. 33-100.

6. Федорчук В. В. Вероятностные меры в топологии // УМН. 1991. Т. 46. № 1. С. 41-80.

7. Банах Т. О. Топология пространств вероятностных мер, I // Математичні студії. 1995. № 5. C. $65-87$.

8. Банах Т. О. Топология пространств вероятностных мер, II // Математичні студії. 1995. № 5. C. $88-106$.

9. Банах Т. О., Коти Р. Топологическая классификация пространств вероятностных мер коаналитических множеств // Матем. заметки. 1994. Т. 55. № 1. С. 10-19.

10. Банах Т. О. Топологическая классификация пространств вероятностных мер на проективных пространствах // Матем. заметки. 1997. Т. 61. № 3. С. 441-444.

11. Радул Т. Н. О некоторых представлениях псевдовнутренности гильбертова куба в пространствах вероятностных мер // Вестник МГУ. Сер. І. Матем., мех. 1993. № 3. С. 79-81.

12. Жураев Т. Ф. Пространство всех вероятностных мер с конечными носителями гомеоморфно бесконечномерному линейному пространству // Общая топология. Пространства и отображения. М.: Изд-во МГУ, 1989. С. 66-70.

13. Toruńczyk $H$. Concerning locally homotopy negligible sets and characterization of $l_{2}$-manifolds // Fund. Math. 1978. V. 101. P. 93-110.

14. Bessaga C., Petczyński A. Selected topics in infinite-dimensional topology. Warsaw: PWN, 1975.

15. van Mill J. Infinite dimensional topology: prerequisites and introduction. Amsterdam: North-Holland, 1989.

16. Banakh T., Radul T., Zarichnyi M. Absorbing sets in infinite-dimensional manifolds. Lviv: VNTL Publishers, 1996.

17. Банах Т. О. О топологии конструкции Милнора универсального $G$-расслоения // Сиб. матем. журн. 1992. Т. 33. № 1. С. 16-25. 
18. Куратовский К. Топология, І. М.: Мир, 1966.

19. Curtis D. W., Dobrowolski T., Mogilski J. Some applications of the topological characterizations of the sigma-compact spaces $l_{f}^{2}$ and $\Sigma / /$ Trans. Amer. Math. Soc. 1984. V. 284. P. 837-846.

20. Федерер Г. Геометрическая теория меры. М.: Наука, 1987.

21. Kechris A.S. Classical descriptive set theory. Graduate texts in Mathematics. V. 156. New York: Springer-Verlag, 1995.

22. Banakh T. The strongly universal property in convex sets // Математичні Студіiі (to appear).

23. Toruńczyk H. Absolute retracts as factors of normed linear spaces // Fund. Math. 1974. V. 86. P. 53-67.

24. Toruńczyk H. A correction of two papers concerning Hilbert manifolds // Fund. Math. 1985. V. 125. P. 89-93.

25. Федорчук B. В., Филиппов В. В. Общая топология. Основные конструкции. М.: Изд-во МГУ, 1988.

26. Энгелькинг Р. Общая топология. М.: Мир, 1986.

27. West J. E. The ambient homeomorphy of an incomplete subspace of incomplete subspace of infinite-dimensional Hilbert spaces // Pacific J. Math. 1970. V. 34. № 1. P. 257-267.

28. Чепмән T. Лекции о $Q$-многообразиях. М.: Мир, 1981.

Львовский университет им. Ив. Франко

Поступила в редакцию

30.10 .1995 